\title{
THE SHARP QUANTITATIVE ISOCAPACITARY INEQUALITY
}

\author{
GUIDO DE PHILIPPIS, MICHELE MARINI, AND EKATERINA MUKOSEEVA
}

Abstract. We prove a sharp quantitative form of the classical isocapacitary inequality. Namely, we show that the difference between the capacity of a set and that of a ball with the same volume bounds the square of the Fraenkel asymmetry of the set. This provides a positive answer to a conjecture of Hall, Hayman, and Weitsman (J. d' Analyse Math. '91).

\section{INTRODUCTION}

1.1. Background. Let $\Omega \subset \mathbb{R}^{N}, N \geq 3$ be an open set. We define the absolute capacity of $\Omega$ as

$$
\operatorname{cap}(\Omega)=\inf _{u \in C_{c}^{\infty}\left(\mathbb{R}^{N}\right)}\left\{\int_{\mathbb{R}^{N}}|\nabla u|^{2} d x: u \geq 1 \text { on } \Omega\right\} .
$$

Moreover, for $\Omega \subset B_{R}$ ( $B_{R}$ the ball of radius $R$ centered at the origin) we denote by $\operatorname{cap}_{R}(\Omega)$ the relative capacity of $\Omega$ with respect to $B_{R}$ defined as

$$
\operatorname{cap}_{R}(\Omega)=\inf _{u \in C_{c}^{\infty}\left(B_{R}\right)}\left\{\int_{B_{R}}|\nabla u|^{2} d x: u \geq 1 \text { on } \Omega\right\} .
$$

It is easy to see that for problem (1.1) (resp. (1.2)) there exists a unique function ${ }^{1} u \in$ $D^{1,2}\left(\mathbb{R}^{N}\right)$ (resp. $u_{R} \in W_{0}^{1,2}\left(B_{R}\right)$ ) called capacitary potential of $\Omega$ such that

$$
\int_{\mathbb{R}^{N}}|\nabla u|^{2}=\operatorname{cap}(\Omega) \quad\left(\operatorname{resp} \cdot \int_{B_{R}}\left|\nabla u_{R}\right|^{2}=\operatorname{cap}_{R}(\Omega)\right) .
$$

Moreover, they satisfy the Euler-Lagrange equations:

$$
\left\{\begin{array} { l } 
{ \Delta u = 0 \text { in } \overline { \Omega } ^ { c } } \\
{ u = 1 \text { on } \partial \Omega } \\
{ u ( x ) \rightarrow 0 \text { as } x \rightarrow 0 }
\end{array} \quad \left\{\begin{array}{l}
\Delta u_{R}=0 \text { in } B_{R} \backslash \bar{\Omega} \\
u_{R}=1 \text { on } \partial \Omega \\
u_{R}=0 \text { on } \partial B_{R} .
\end{array}\right.\right.
$$

The well-known isocapacitary inequality (resp. relative isocapacitary inequality) asserts that, among all sets with given volume, balls (resp. ball centered at the origin) have the smallest possible capacity, namely

$$
\operatorname{cap}(\Omega)-\operatorname{cap}\left(B_{r}\right) \geq 0 \quad\left(\operatorname{resp} . \operatorname{cap}_{\mathrm{R}}(\Omega)-\operatorname{cap}_{\mathrm{R}}\left(B_{r}\right) \geq 0\right) .
$$

Here $r$ is such that $\left|B_{r}\right|=|\Omega|$, where $|\cdot|$ denotes the Lebesgue measure.

\footnotetext{
2010 Mathematics Subject Classification. 49R05 (47A75 49Q20).

Key words and phrases. Isocapacitary inequality, stability estimates, Fraenkel asymmetry.

${ }^{1}$ Here and in the sequel, $D^{1,2}\left(\mathbb{R}^{N}\right)$ denotes the closure of $C_{c}^{\infty}\left(\mathbb{R}^{N}\right)$ with respect to the homogeneous Sobolev norm:

$$
\|u\|_{\dot{W}^{1,2}}:=\|\nabla u\|_{L^{2}}
$$
}

see [EG15, Section 4.7] and [LL97, Chapter 8] 
The proof is an easy combination of Schwarz symmetrization with Pólya-Szegö principle. Indeed, let $\Omega$ be an open set and let $u$ be its capacitary potential. Schwarz symmetrization provides us with a radially symmetric function $u^{*}$ such that, for every $t \in \mathbb{R}$,

$$
|\{x: u(x)>t\}|=\left|\left\{x: u^{*}(x)>t\right\}\right| .
$$

We use $u^{*}$ as a test function for the set $\left\{x: u^{*}(x)=1\right\}=B_{r}$ and we note that (1.4) yields that $\left.\left|B_{r}\right|=|\Omega|\right)$. Hence

$$
\operatorname{cap}\left(B_{r}\right) \leq \int_{\mathbb{R}^{N}}\left|\nabla u^{*}\right|^{2} d x \leq \int_{\mathbb{R}^{N}}|\nabla u|^{2} d x=\operatorname{cap}(\Omega) \quad|\Omega|=\left|B_{r}\right|,
$$

where the second inequality follows by Pólya-Szegö principle. The very same argument applies to the relative isocapacitary inequality.

Inequalities (1.3) are rigid, namely, equality is attained only when $\Omega$ coincides with a ball, up to a set of zero capacity. For the case of the relative isocapacitary inequality $\Omega$ must instead coincide with a centered ball, since this latter notion of capacity is not invariant under translations.

It is natural to wonder whether these inequalities are also stable, that is $\Omega \rightarrow B_{r}$, whenever $\operatorname{cap}(\Omega) \rightarrow \operatorname{cap}\left(B_{r}\right)$. In particular, one aims to a (possibly sharp) quantitative enhancement of inequalities (1.3) by replacing their right-hand side with some function of the distance of $\Omega$ from the set of balls.

As we shall explain in the following sections, the answer is positive, and a good choice of distance is the so-called Fraenkel asymmetry.

Definition 1.1. Let $\Omega$ be an open set. The Fraenkel asymmetry of $\Omega, \mathcal{A}(\Omega)$, is defined as:

$$
\mathcal{A}(\Omega)=\inf \left\{\frac{|\Omega \Delta B|}{|B|}: B \text { is a ball with the same volume as } \Omega\right\} .
$$

To the best of our knowledge, the first results in this direction appeared in [HHW91] where they considered the case of simply connected planar sets ${ }^{2}$ and of convex sets in general dimension. In the same paper the authors conjecture the validity of the following inequality:

Conjecture 1.2 ([HHW91]). Let $N \geq 3$. There exists a constant $c=c(N)$ such that for any open set $\Omega$ the following inequality holds:

$$
\frac{\operatorname{cap}(\Omega)-\operatorname{cap}\left(B_{r}\right)}{r^{N-2}} \geq c \mathcal{A}(\Omega)^{2} .
$$

Note that by testing the inequality on ellipsoids with eccentricity $\varepsilon$ one easily sees that the exponent 2 can not be replaced by any smaller number.

A positive answer to the above conjecture in dimension 2 has been given by Hansen and Nadirashvili in [HN92]. For general dimension, the best known result is due to Fusco, Maggi, and Pratelli in [FMP09] where they prove the following:

Theorem 1.3 ([FMP09]). There exists a constant $c=c(N)$ such that, for any open set $\Omega$

$$
\frac{\operatorname{cap}(\Omega)-\operatorname{cap}\left(B_{r}\right)}{r^{N-2}} \geq c \mathcal{A}(\Omega)^{4} .
$$

In this paper we provide a positive answer to Conjecture 1.2 in every dimension and to its version for the relative capacity.

\footnotetext{
${ }^{2}$ Note that for $N=2$ the infimum (1.1) is 0 and one has to use the notion of logarithmic capacity.
} 
1.2. Main result. The following is the main result of the paper, note that by the scaling $\operatorname{cap}(\lambda \Omega)=\lambda^{N-2} \operatorname{cap}(\Omega)$, we can also get the analogous result for $\Omega$ with arbitrary volume.

Theorem 1.4. Let $\Omega$ be an open set such that $|\Omega|=\left|B_{1}\right|$. Then

(A) if $\Omega$ is contained in $B_{R}$, there exists a constant $c_{1}=c_{1}(N, R)$ such that the following inequality holds:

$$
\operatorname{cap}_{R}(\Omega)-\operatorname{cap}_{R}\left(B_{1}\right) \geq c_{1}(N, R)\left|\Omega \Delta B_{1}\right|^{2} .
$$

(B) there exists a constant $c_{2}=c_{2}(N)$ such that the following inequality holds:

$$
\operatorname{cap}(\Omega)-\operatorname{cap}\left(B_{1}\right) \geq c_{2}(N) \mathcal{A}(\Omega)^{2} .
$$

Note that in the above theorem, in the case of the absolute capacity one bound the distance of $\Omega$ from the set of balls, while in the case of the relative capacity one bounds the distance from the ball centered at the origin but the constant is $R$ dependent. Indeed in the former case all balls have the same capacity (due to the translation invariance of the problem) and thus in order to obtain a quantitative improvement, one has to measure the distance from the set of all minimizers. On the contrary, for the relative capacity, the ball centered at the origin is the only minimizer. Since

$$
\lim _{R \rightarrow+\infty} \operatorname{cap}_{R}(\Omega)=\operatorname{cap}(\Omega),
$$

it is clear that the constant in (B) above needs to depend on $R$. This can also be inferred by the study of the linearized problem, see Section 2.2 below. We also remark that, as it will be clear from the proof, in the case of the relative capacity one can replace $\left|\Omega \Delta B_{1}\right|$ with the bigger quantity $\alpha_{R}(\Omega)$ defined in Section 2.2 below.

1.3. Strategy of the proof and structure of the paper. Since the isocapacitary inequality is a consequence of the isoperimetric inequality, a reasonable strategy to obtain a quantitative improvement would be to rely on a quantitative isoperimetric inequality. This was indeed the strategy used in [FMP09] where they rely on the quantitative isoperimetric inequality established in [FMP08]. However, although the inequality proved in [FMP08] is sharp, in order to combine it with the Schwarz symmetrization procedure, it seems unavoidable to lose some exponent and to obtain a result in line with the one in [FMP09].

Here we instead rely on the techniques developed by the first author with Brasco and Velichkov in [BDPV15] to obtain a quantitative form of the Faber-Krahn inequality (see also [BDP17] and references therein for a survey on these type of results). The proof is based on the Selection Principle, introduced by Cicalese and Leonardi in [CL12] to give a new proof of the sharp quantitative isoperimetric inequality, combined with the regularity estimates for free boundary problems obtained by Alt and Caffarelli in [AC81]. As in [BDPV15], one of the key technical tools is to replace the Fraenkel asymmetry (which roughly resembles a $L^{1}$ type norm) with a smoother (and stronger) version inspired by the distance among sets first used by Almgren Taylor and Wang in [ATW93] which resembles an $L^{2}$ type norm, see Section 2.2 for the exact definition.

We conclude this introduction by giving an account of the main steps of the proof and of the structure of the paper:

The main step consists in proving Theorem 1.4 for a priori bounded sets in the regime of small asymmetry. Arguing by contradiction one obtains a sequence of sets contradicting the stability inequality with any given constant $c>0$. In Sections 3 and 4 we use this sequence to construct an improved contradicting sequence which solves a variational problem.

In Section 5, we exploit the regularity theory of [AC81] to show that this new sequence consists of smooth nearly spherical sets, for which the desired estimate is proved in Section 2, via a Fuglede type computation [Fug89]. In Section 6, we show how one can reduce to a 
priori bounded domains for the case of the absolute capacity. Eventually, in Section 7 we combine all the steps to prove Theorem 1.4.

Acknowledgements. The work of the authors is supported by the INDAM-grant "Geometric Variational Problems".

\section{Fuglede's Computation}

As explained in the introduction it is convenient to introduce a smoothed version of the Fraenkel asymmetry. Roughly speaking, while $\mathcal{A}(\Omega)$ represents an $L^{1}$ norm, $\alpha(\Omega)$ represents an $L^{2}$ norm, see (iii) in Lemma 2.3 below and the discussion in [BDPV15, Introduction].

Definition 2.1. Let $\Omega$ be an open set in $\mathbb{R}^{N}$. Then we define the asymmetry $\alpha$ in the following way:

(A)

$$
\alpha_{R}(\Omega)=\int_{\Omega \Delta B_{1}}|1-| x|| d x
$$

$$
\alpha(\Omega)=\int_{\Omega \Delta B_{1}\left(x_{\Omega}\right)}|1-| x-x_{\Omega}|| d x .
$$

Here $x_{\Omega}$ denotes the barycenter of $\Omega$, namely $x_{\Omega}=f_{\Omega} x d x$.

Since most of the argument will be similar for the relative and for the absolute capacity, let us also introduce the following notational convention:

Notation 2.2. Whenever possible, we will write $\alpha_{*}, \operatorname{cap}_{*}$, etc. instead of $\alpha / \alpha_{R}$, cap $/ \operatorname{cap}_{R}$ or other notions that will come along. The convention is that $*$ denotes the same thing $(R$ or the absence of it) throughout the equation or the computation where it appears.

The next Lemma collects the main properties of $\alpha$, the proof is identical to the one of [BDPV15, Lemma 4.2] and it is left to the reader.

Lemma 2.3. Let $\Omega \subset \mathbb{R}^{n}$, then

(i) There exists a constant $c=c(N)$ such that

(A)

$$
\alpha_{R}(\Omega) \geq c\left|\Omega \Delta B_{1}\right|^{2}
$$

for any open set $\Omega \subset B_{R}$;

for any open set $\Omega$.

$$
\alpha(\Omega) \geq c\left|\Omega \Delta B_{1}\left(x_{\Omega}\right)\right|^{2}
$$

(ii) There exists a constant $C=C(R)$ such that

$$
\left|\alpha_{*}\left(\Omega_{1}\right)-\alpha_{*}\left(\Omega_{2}\right)\right| \leq C\left|\Omega_{1} \Delta \Omega_{2}\right|
$$

for any $\Omega_{1}, \Omega_{2} \subset B_{R}$. In particular, if $1_{\Omega_{k}} \rightarrow 1_{\Omega}$ in $L^{1}\left(B_{R}\right)$ then $\alpha_{*}\left(\Omega_{k}\right) \rightarrow \alpha_{*}(\Omega)$.

(iii) There exist constants $C=C(N), \delta=\delta(N)$ such that for every nearly spherical set (see Definition 2.4 below) $\Omega$ with $\|\phi\|_{\infty} \leq \delta$ (and $x_{\Omega}=0$ in the case of $\alpha$ )

$$
\alpha_{*}(\Omega) \leq C\|\phi\|_{L^{2}\left(\partial B_{1}\right)}^{2} .
$$

We now prove the validity of the quantitative isocapacitary inequality for sets close to the unit ball. More precisely, we are going to prove Theorem 1.4 for nearly spherical sets which are defined below. The proof is based on second variation argument as in [Fug89]. 
Definition 2.4. An open bounded set $\Omega \subset \mathbb{R}^{N}$ is called nearly spherical of class $C^{2, \gamma}$ parametrized by $\varphi$, if there exists $\varphi \in C^{2, \gamma}$ with $\|\varphi\|_{L^{\infty}}<\frac{1}{2}$ such that

$$
\partial \Omega=\left\{(1+\varphi(x)) x: x \in \partial B_{1}\right\} .
$$

Let us also introduce the following definition:

Definition 2.5. Given a function $\varphi: \partial B_{1} \rightarrow \mathbb{R}$ we define

(A) $H_{R}(\varphi) \in W_{0}^{1,2}\left(B_{R}\right)$ as the solution to

$$
\left\{\begin{array}{l}
\Delta H_{R}(\varphi)=0 \text { in } B_{R} \backslash B_{1} \\
H_{R}(\varphi)=\varphi \text { on } \partial B_{1} \\
H_{R}(\varphi)=0 \text { on } \partial B_{R}
\end{array}\right.
$$

(B) $H(\varphi) \in D^{1,2}\left(\mathbb{R}^{N}\right)$ as the solution to

$$
\left\{\begin{array}{l}
\Delta H(\varphi)=0 \text { in } B_{1}^{c} \\
H(\varphi)=\varphi \text { on } \partial B_{1} \\
H(\varphi)(x) \rightarrow 0 \text { as } x \rightarrow \infty
\end{array}\right.
$$

2.1. Second variation. We now compute the second order expansion of the capacity of a nearly spherical set. Note that the remainder term is multiplied by a higher order norm. This is precisely the reason why we will need to use the Selection Principle in the proof of Theorem 1.4.

Lemma 2.6. Given $\gamma \in(0,1]$, there exists $\delta=\delta(N, \gamma)>0$ and a modulus of continuity $\omega$ such that for every nearly spherical set $\Omega$ parametrized by $\varphi$ with $\|\varphi\|_{C^{2, \gamma}\left(\partial B_{1}\right)}<\delta$ and $|\Omega|=\left|B_{1}\right|$, we have

$$
\operatorname{cap}_{*}(\Omega) \geq \operatorname{cap}_{*}\left(B_{1}\right)+\frac{1}{2} \partial^{2} \operatorname{cap}_{*}\left(B_{1}\right)[\varphi, \varphi]-\omega\left(\|\varphi\|_{C^{2, \gamma}}\right)\|\varphi\|_{H^{\frac{1}{2}}\left(\partial B_{1}\right)}^{2},
$$

where

(A)

$$
\partial^{2} \operatorname{cap}_{R}\left(B_{1}\right)[\varphi, \varphi]:=2 \frac{(N-2)^{2}}{1-R^{-(N-2)}}\left(\int_{B_{R} \backslash B_{1}}\left|\nabla H_{R}(\varphi)\right|^{2} d x-(N-1) \int_{\partial B_{1}} \varphi^{2} d \mathcal{H}^{N-1}\right) ;
$$

$$
\partial^{2} \operatorname{cap}\left(B_{1}\right)[\varphi, \varphi]:=2(N-2)^{2}\left(\int_{B_{1}^{c}}|\nabla H(\varphi)|^{2} d x-(N-1) \int_{\partial B_{1}} \varphi^{2} d \mathcal{H}^{N-1}\right) .
$$

To prove it, let us first introduce a technical lemma.

Lemma 2.7. Given $\gamma \in(0,1]$ there exists $\delta=\delta(N, \gamma)>0$ and a modulus of continuity $\omega$ such that for every nearly spherical set $\Omega$ parametrized by $\varphi$ with $\|\varphi\|_{C^{2, \gamma}\left(\partial B_{1}\right)}<\delta$ and $|\Omega|=\left|B_{1}\right|$, we can find an autonomous vector field $X_{\varphi}$ for which the following holds true:

(i) $\operatorname{div} X_{\varphi}=0$ in a $\delta$-neighborhood of $\partial B_{1}$;

(ii) $X_{\varphi}=0$ outside a $2 \delta$-neighborhood of $\partial B_{1}$;

(iii) if $\Phi_{t}:=\Phi(t, x)$ is the flow of $X_{\varphi}$, i.e.

$$
\partial_{t} \Phi_{t}=X_{\varphi}\left(\Phi_{t}\right), \quad \Phi_{0}(x)=x
$$

then $\Phi_{1}\left(\partial B_{1}\right)=\partial \Omega$ and $\left|\Phi_{t}\left(B_{1}\right)\right|=\left|B_{1}\right|$ for all $t \in[0,1]$;

(iv) $-\left\|\Phi_{t}-I d\right\|_{C^{2, \gamma}} \leq \omega\left(\|\varphi\|_{C^{2, \gamma}\left(\partial B_{1}\right)}\right)$ for every $t \in[0,1]$, 
- $\left\|\varphi-\left(X_{\varphi} \cdot \nu_{B_{1}}\right)\right\|_{H^{\frac{1}{2}\left(\partial B_{1}\right)}} \leq \omega\left(\|\varphi\|_{L^{\infty}\left(\partial B_{1}\right)}\right)\|\varphi\|_{H^{\frac{1}{2}\left(\partial B_{1}\right)}}$,

- $(X \cdot x) \circ \Phi_{t}-X \cdot \nu_{B_{1}}=\left(X \cdot \nu_{B_{1}}\right) \psi_{t}, x \in \partial B_{1}$, where $\left\|\psi_{t}\right\|_{C^{2, \gamma}\left(\partial B_{1}\right)} \leq \omega\left(\|\varphi\|_{C^{2, \gamma}\left(\partial B_{1}\right)}\right)$.

Proof. Take the same vector field as in Appendix of [BDPV15] and multiply it by a cut-off function.

Proof of Lemma 2.6. Now set $\Omega_{t}:=\Phi_{t}\left(B_{1}\right)$ and let $u_{t}$ be the capacitary potential of $\Omega_{t}$. We define

$$
c_{*}(t):=\operatorname{cap}_{*}\left(\Omega_{t}\right)=\left\{\begin{array}{l}
\int_{B_{R} \backslash \Omega_{t}}\left|\nabla u_{t}\right|^{2} d x \text { in the case of relative capacity; } \\
\int_{\Omega_{t}^{c}}\left|\nabla u_{t}\right|^{2} d x \text { in the case of full capacity. }
\end{array}\right.
$$

It is easy to see that $t \mapsto u_{t}$ is differentiable, see [Dam02], and that its derivative $u_{t}$ satisfies

(A)

$$
\left\{\begin{array}{l}
\Delta \dot{u}_{t}=0 \text { in } B_{R} \backslash \Omega_{t} \\
\dot{u_{t}}=-\nabla u_{t} \cdot X_{\varphi} \text { on } \partial \Omega_{t} \\
\dot{u}_{t}=0 \text { on } \partial B_{R}
\end{array}\right.
$$

(B)

$$
\left\{\begin{array}{l}
\Delta \dot{u}_{t}=0 \text { in } \Omega_{t}^{c} \\
\dot{u}_{t}=-\nabla u_{t} \cdot X_{\varphi} \text { on } \partial \Omega_{t} \\
\dot{u}_{t}(x) \rightarrow 0 \text { as } x \rightarrow 0
\end{array}\right.
$$

Using Hadamard formula, we compute:

$$
\frac{1}{2} c_{R}^{\prime}(t)=\int_{B_{R} \backslash \Omega_{t}} \nabla u_{t} \cdot \nabla \dot{u}_{t} d x+\frac{1}{2} \int_{\partial \Omega_{t}}\left|\nabla u_{t}\right|^{2} X_{\varphi} \cdot \nu_{\Omega_{t}} d \mathcal{H}^{N-1},
$$

where $\nu_{\Omega_{t}}$ is the inward normal to $\partial \Omega_{t}$. Now we recall that $u_{t}$ is harmonic in $B_{R} \backslash \Omega_{t}$ and we use the boundary conditions for $\dot{u}_{t}$ to get

$$
\begin{aligned}
\frac{1}{2} c_{R}^{\prime}(t) & =\int_{B_{R} \backslash \Omega_{t}} \operatorname{div}\left(\dot{u}_{t} \nabla u_{t}\right) d x+\frac{1}{2} \int_{\partial \Omega_{t}}\left|\nabla u_{t}\right|^{2} X_{\varphi} \cdot \nu_{\Omega_{t}} d \mathcal{H}^{N-1} \\
& =\int_{\partial \Omega_{t}} \dot{u_{t}} \nabla u_{t} \cdot \nu_{\Omega_{t}} d \mathcal{H}^{N-1}+\frac{1}{2} \int_{\partial \Omega_{t}}\left|\nabla u_{t}\right|^{2} X_{\varphi} \cdot \nu_{\Omega_{t}} d \mathcal{H}^{N-1} \\
& =\int_{\partial \Omega_{t}}\left(-\nabla u_{t} \cdot X_{\varphi}\right) \nabla u_{t} \cdot \nu_{\Omega_{t}} d \mathcal{H}^{N-1}+\frac{1}{2} \int_{\partial \Omega_{t}}\left|\nabla u_{t}\right|^{2} X_{\varphi} \cdot \nu_{\Omega_{t}} d \mathcal{H}^{N-1} .
\end{aligned}
$$

We know that $u_{t}$ is identically 1 on $\partial \Omega_{t}$ and smaller than 1 outside, hence (recall that $\nu_{\partial \Omega_{t}}$ denotes the ineer normal)

$$
\nabla u_{t}=\left|\nabla u_{t}\right| \nu_{\partial \Omega_{t}} \text { on } \partial \Omega_{t} .
$$

Therefore,

$$
\begin{aligned}
\frac{1}{2} c_{R}^{\prime}(t) & =\int_{\partial \Omega_{t}}-\left|\nabla u_{t}\right|^{2} X_{\varphi} \cdot \nu_{\Omega_{t}} d \mathcal{H}^{N-1}+\frac{1}{2} \int_{\partial \Omega_{t}}\left|\nabla u_{t}\right|^{2} X_{\varphi} \cdot \nu_{\Omega_{t}} d \mathcal{H}^{N-1} \\
& =-\frac{1}{2} \int_{\partial \Omega_{t}}\left|\nabla u_{t}\right|^{2} X_{\varphi} \cdot \nu_{\Omega_{t}} d \mathcal{H}^{N-1}=-\frac{1}{2} \int_{B_{R} \backslash \Omega_{t}} \operatorname{div}\left(\left|\nabla u_{t}\right|^{2} X_{\varphi}\right) d x
\end{aligned}
$$


We proceed now with the second derivative, using again Hadamard's formula and recalling that $X$ is autonomous and divergence-free in a neighborhood of $\partial B_{1}$ (hence, on $\partial \Omega_{t}$ ).

$$
\begin{aligned}
\frac{1}{2} c_{R}^{\prime \prime}(t) & =-\frac{1}{2} \int_{B_{R} \backslash \Omega_{t}} \operatorname{div}\left(\frac{\partial}{\partial t}\left|\nabla u_{t}\right|^{2} X_{\varphi}\right) d x-\frac{1}{2} \int_{\partial \Omega_{t}} \operatorname{div}\left(\left|\nabla u_{t}\right|^{2} X_{\varphi}\right)\left(X_{\varphi} \cdot \nu_{\Omega_{t}}\right) d \mathcal{H}^{N-1} \\
& =-\int_{\partial \Omega_{t}}\left(\nabla u_{t} \cdot \nabla \dot{u}_{t}\right) X_{\varphi} \cdot \nu_{\Omega_{t}} d \mathcal{H}^{N-1}-\frac{1}{2} \int_{\partial \Omega_{t}}\left(\nabla\left|\nabla u_{t}\right|^{2} \cdot X_{\varphi}\right)\left(X_{\varphi} \cdot \nu_{\Omega_{t}}\right) d \mathcal{H}^{N-1} \\
& =\int_{\partial \Omega_{t}} \dot{u}_{t} \nabla \dot{u}_{t} \cdot \nu_{\Omega_{t}} d \mathcal{H}^{N-1}-\int_{\partial \Omega_{t}}\left(X_{\varphi} \cdot \nu_{\Omega_{t}}\right)\left(\nabla^{2} u_{t}\left[\nabla u_{t}\right] \cdot X_{\varphi}\right) d \mathcal{H}^{N-1} \\
& =\int_{B_{R} \backslash \Omega_{t}}\left|\nabla \dot{u}_{t}\right|^{2} d x-\int_{\partial \Omega_{t}}\left(X_{\varphi} \cdot \nu_{\Omega_{t}}\right)\left(\nabla^{2} u_{t}\left[\nabla u_{t}\right] \cdot X_{\varphi}\right) d \mathcal{H}^{N-1}
\end{aligned}
$$

Note that in the second to last equality we have used (2.1) and the boundary condition for $\dot{u}_{t}$. Now since $u_{t}$ is constant on $\partial \Omega_{t}$, we get

$$
0=\Delta u_{t}=\left|\nabla u_{t}\right| \mathscr{H}_{\partial \Omega_{t}}+\nabla^{2}\left[\nu_{\Omega_{t}}\right] \cdot \nu_{\Omega_{t}} \text { on } \partial \Omega_{t},
$$

where $\mathscr{H}_{\partial \Omega_{t}}$ is the mean curvature of $\partial \Omega_{t}$ with respect to the inward normal to $\partial \Omega_{t}$. Taking this into account and denoting $X^{\tau}=X_{\varphi}-\left(X_{\varphi} \cdot \nu_{\Omega_{t}}\right) \nu_{\Omega_{t}}$ on $\partial \Omega_{t}$, we get

$$
\begin{aligned}
\frac{1}{2} c_{R}^{\prime \prime}(t)= & \int_{B_{R} \backslash \Omega_{t}}\left|\nabla \dot{u}_{t}\right|^{2} d x \\
& -\int_{\partial \Omega_{t}}\left(X_{\varphi} \cdot \nu_{\Omega_{t}}\right)\left(\nabla^{2} u_{t}\left[\left|\nabla u_{t}\right| \nu_{\Omega_{t}}\right] \cdot\left(\left(X_{\varphi} \cdot \nu_{\Omega_{t}}\right) \nu+X^{\tau}\right)\right) d \mathcal{H}^{N-1} \\
= & \int_{B_{R} \backslash \Omega_{t}}\left|\nabla u_{t}\right|^{2} d x+\int_{\partial \Omega_{t}}\left(X_{\varphi} \cdot \nu_{\Omega_{t}}\right)^{2}\left|\nabla u_{t}\right|^{2} \mathscr{H}_{\partial \Omega_{t}} d \mathcal{H}^{N-1} \\
& -\int_{\partial \Omega_{t}}\left(X_{\varphi} \cdot \nu_{\Omega_{t}}\right)\left(\nabla^{2} u_{t}\left[\nabla u_{t}\right] \cdot X^{\tau}\right) d \mathcal{H}^{N-1} .
\end{aligned}
$$

Now we wish to calculate $c_{R}^{\prime \prime}(0)$. We use that

- $\mathscr{H}_{\partial B_{1}}=-(N-1)$

- $X^{\tau}=0$ on $\partial B_{1}$;

- $u_{0}=u_{B_{1}}=\frac{|x|^{-(N-2)}-R^{-(N-2)}}{1-R^{-(N-2)}}$ in $B_{R} \backslash B_{1}$;

- $u_{0}=H_{R}\left(-X_{\varphi} \cdot \nabla u_{0}\right)$.

$$
\begin{aligned}
\frac{1}{2} c_{R}^{\prime \prime}(0) & =\int_{B_{R} \backslash B_{1}}\left|\nabla H_{R}\left(-X_{\varphi} \cdot \nabla u_{0}\right)\right|^{2} d x-(N-1) \int_{\partial B_{1}}\left(X_{\varphi} \cdot \nu_{B_{1}}\right)^{2}\left|\nabla u_{0}\right|^{2} d \mathcal{H}^{N-1} \\
& =\frac{(N-2)^{2}}{1-R^{-(N-2)}}\left(\int_{B_{R} \backslash B_{1}}\left|\nabla H_{R}\left(X_{\varphi} \cdot \nu_{B_{1}}\right)\right|^{2} d x-(N-1) \int_{\partial B_{1}}\left(X_{\varphi} \cdot \nu_{B_{1}}\right)^{2} d \mathcal{H}^{N-1}\right)
\end{aligned}
$$

As for the case of full capacity, the same computations apply with minor changes, obtaining

$$
\frac{1}{2} c^{\prime \prime}(0)=(N-2)^{2}\left(\int_{B_{1}^{c}}\left|\nabla H_{R}\left(X_{\varphi} \cdot \nu_{B_{1}}\right)\right|^{2} d x-(N-1) \int_{\partial B_{1}}\left(X_{\varphi} \cdot \nu_{B_{1}}\right)^{2} d \mathcal{H}^{N-1}\right),
$$

which formally corresponds to sending $R \rightarrow \infty$ in the formula for $c_{R}^{\prime \prime}$. Since balls minimize the capacity we also have that $c_{*}^{\prime}(0)=0$. Writing

$$
\operatorname{cap}_{*}(\Omega)=c_{*}(1)=c_{*}(0)+\frac{1}{2} c_{*}^{\prime \prime}(0)+\int_{0}^{1}(1-t)\left(c_{*}^{\prime \prime}(t)-c_{*}^{\prime \prime}(0)\right) d t,
$$


one can now exploit Lemma 2.7 and perform the very same computations as in [BDPV15, Lemma A.2] to conclude.

2.2. Inequality for nearly spherical sets. We now establish a quantitative inequality for nearly spherical sets in the spirit of those established by Fuglede in [Fug89], compare with [BDPV15, Section 3].

Theorem 2.8. There exists $\delta=\delta(N), c=c(N, R)\left(c=c(N)\right.$ for the capacity in $\left.\mathbb{R}^{N}\right)$ such that if $\Omega$ is a nearly spherical set of class $C^{2, \gamma}$ parametrized by $\varphi$ with $\|\varphi\|_{C^{2, \gamma}} \leq \delta,|\Omega|=$ $\left|B_{1}\right|$ (and $x_{\Omega}=0$ for the case of the capacity in $\mathbb{R}^{N}$ ), then

$$
\operatorname{cap}_{*}(\Omega)-\operatorname{cap}_{*}\left(B_{1}\right) \geq c\|\varphi\|_{H^{\frac{1}{2}}\left(\partial B_{1}\right)}^{2},
$$

where

$$
\|\varphi\|_{H^{\frac{1}{2}\left(\partial B_{1}\right)}}^{2}:=\int_{\partial B_{1}} \varphi^{2} d \mathcal{H}^{N-1}+\int_{B_{1}^{c}}\left|\nabla H_{*}(\varphi)\right|^{2} d x,
$$

where the second integral is intended on $B_{R} \backslash B_{1}$ if $*=R$.

Remark 2.9. Note that by Lemma 2.3 (i),(iii) this theorem gives us Theorem 1.4 for nearly spherical sets.

Proof. We essentially repeat the proof of the Theorem 3.3 in [BDPV15]. First, we show that $\int_{\partial B_{1}} \varphi$ is small. Indeed, we know that

$$
\begin{aligned}
\left|B_{1}\right|=|\Omega| & =\int_{\partial B_{1}} \frac{(1+\varphi(x))^{N}}{N} d \mathcal{H}^{N-1} \\
& =\left|B_{1}\right|+\int_{\partial B_{1}} \varphi(x) d \mathcal{H}^{N-1}+\int_{\partial B_{1}} \sum_{i=2}^{N}\left(\begin{array}{c}
N \\
i
\end{array}\right) \frac{\varphi(x)^{i}}{N} d \mathcal{H}^{N-1} .
\end{aligned}
$$

Hence,

$$
\begin{aligned}
\left|\int_{\partial B_{1}} \varphi(x) d \mathcal{H}^{N-1}\right| & =\left|\int_{\partial B_{1}} \sum_{i=2}^{N}\left(\begin{array}{c}
N \\
i
\end{array}\right) \frac{\varphi(x)^{i}}{N} d \mathcal{H}^{N-1}\right| \\
& \leq C(N) \int_{\partial B_{1}} \varphi(x)^{2} d \mathcal{H}^{N-1} \leq C(N) \delta\|\varphi\|_{L^{2}}
\end{aligned}
$$

Moreover, for the case of the absolute capacity, also $\int_{\partial B_{1}} x_{i} \varphi$ is small. Indeed, using that the barycenter of $\Omega$ is at the origin, we get

$$
\left|\int_{\partial B_{1}} x_{i} \varphi(x) d \mathcal{H}^{N-1}\right| \leq \int_{\partial B_{1}} \sum_{i=2}^{N}\left(\begin{array}{c}
N \\
i
\end{array}\right)\left|\frac{\varphi(x)^{i}}{N+1}\right| d \mathcal{H}^{N-1} \leq C(N) \delta\|\varphi\|_{L^{2}} .
$$

Let us define

(A)

(B)

$$
\mathcal{M}_{\delta}^{R}:=\left\{\xi \in H^{\frac{1}{2}}\left(\partial B_{1}\right):\left|\int_{\partial B_{1}} \xi d \mathcal{H}^{N-1}\right| \leq \delta\|\xi\|_{H^{1 / 2}}\right\}
$$

$$
\mathcal{M}_{\delta}:=\left\{\xi \in H^{\frac{1}{2}}\left(\partial B_{1}\right):\left|\int_{\partial B_{1}} \xi d \mathcal{H}^{N-1}\right|+\left|\int_{\partial B_{1}} x \xi d \mathcal{H}^{N-1}\right| \leq \delta\|\xi\|_{H^{1 / 2}}\right\},
$$


and note that, since $\|\xi\|_{L^{2}} \leq\|\xi\|_{H^{1 / 2}}$, we have just proved that $\varphi$ belongs to $\mathcal{M}_{C \delta}^{*}$.

By Lemma 2.6, for $\delta$ small enough we have

$$
\operatorname{cap}_{*}(\Omega)-\operatorname{cap}_{*}\left(B_{1}\right) \geq \frac{1}{2} \partial^{2} \operatorname{cap}_{*}\left(B_{1}\right)[\varphi, \varphi]-\omega\left(\|\varphi\|_{C^{2, \gamma}}\right)\|\varphi\|_{H^{\frac{1}{2}\left(\partial B_{1}\right)}}^{2} .
$$

So, it is enough to check that

$$
\partial^{2} \operatorname{cap}_{*}\left(B_{1}\right)[\xi, \xi] \geq c\|\xi\|_{H^{\frac{1}{2}\left(\partial B_{1}\right)}}^{2}, \text { for every } \xi \in \mathcal{M}_{\delta}^{*}
$$

for small $\delta$.

Step 1: linearized problem. First, we show that

$$
\partial^{2} \operatorname{cap}_{*}\left(B_{1}\right)[\xi, \xi] \geq c\|\xi\|_{H^{\frac{1}{2}\left(\partial B_{1}\right)}}^{2}, \text { for every } \xi \in \mathcal{M}_{0}^{*}
$$

Note that
(A) $\mathcal{M}_{0}^{R}=\left\{\xi \in H^{\frac{1}{2}}\left(\partial B_{1}\right): \int_{\partial B_{1}} \xi d \mathcal{H}^{N-1}=0\right\}$;
(B) $\mathcal{M}_{0}=\left\{\xi \in H^{\frac{1}{2}}\left(\partial B_{1}\right): \int_{\partial B_{1}} \xi d \mathcal{H}^{N-1}=\int_{\partial B_{1}} x_{i} \xi d \mathcal{H}^{N-1}=0, i=1,2, \ldots, N\right\}$.

We recall that

$$
\partial^{2} \operatorname{cap}_{R}\left(B_{1}\right)[\varphi, \varphi]:=2 \frac{(N-2)^{2}}{1-R^{-(N-2)}}\left(\int_{B_{R} \backslash B_{1}}\left|\nabla H_{R}(\varphi)\right|^{2} d x-(N-1) \int_{\partial B_{1}} \varphi^{2} d \mathcal{H}^{N-1}\right)
$$

$$
\partial^{2} \operatorname{cap}\left(B_{1}\right)[\varphi, \varphi]:=2(N-2)^{2}\left(\int_{B_{1}^{c}}|\nabla H(\varphi)|^{2} d x-(N-1) \int_{\partial B_{1}} \varphi^{2} d \mathcal{H}^{N-1}\right) .
$$

We consider first the case of relative capacity. We need to estimate the quotient

$$
\frac{\int_{B_{R} \backslash B_{1}}\left|\nabla H_{R}(\xi)\right|^{2} d x}{\int_{\partial B_{1}} \xi^{2} d \mathcal{H}^{N-1}}
$$

from below for $\xi \in \mathcal{M}_{0} \backslash\{0\}$. We note that it is the Rayleigh quotient for the operator $\xi \mapsto \nabla H_{R}(\xi) \cdot \nu$. Thus, we need to calculate its eigenvalues. We use spherical functions as a basis of $L^{2}\left(\partial B_{1}\right): \xi=\sum_{m, n} a_{m, n} Y_{m, n}$. We now show that $H\left(Y_{m, n}\right)$ can be written as $R_{m, n}(r) Y_{m, n}(\omega)$ for a suitable function $R_{m, n}(r)$. Indeed, by the equation defining $H\left(Y_{m . n}\right)$ we have check that

$$
\left\{\begin{array}{l}
\Delta\left(R_{m, n}(r) Y_{m, n}(\omega)\right)=0 \text { in } B_{R} \backslash B_{1} \\
R_{m, n}(1) Y_{m, n}=Y_{m, n} \\
R_{m, n}(R) Y_{m, n}=0
\end{array}\right.
$$

Since $\tilde{\Delta} Y_{m, n}=-m(m+N-2) Y_{m, n}$, where $\tilde{\Delta}$ is the Laplace-Beltrami operator, one easily checks that

$$
R_{m, n}(r)=-\frac{1}{R^{2 m+N-2}-1} r^{m}+\left(1+\frac{1}{R^{2 m+N-2}-1}\right) r^{-(N+m-2)}
$$

provides a solution. Hence, the first eigenvalue is zero and corresponds to constants, whereas the first non-zero one is $-R_{1, n}^{\prime}(1)=(N-1)+\frac{1}{R^{N}-1} N$.

For the case of the absolute capacity we estimate the quotient

$$
\frac{\int_{B_{1}^{c}}|\nabla H(\xi)|^{2} d x}{\int_{\partial B_{1}} \xi^{2} d \mathcal{H}^{N-1}}
$$


in an analogous way. The functions $R_{m, n}$ in this case is

$$
R_{m, n}(r)=r^{-(N+m-2)} .
$$

The first eigenvalue is zero and corresponds to constants, the second one is $N-1$ and corresponds to the coordinate functions, the next one is $N$.

Step 2: reducing to $\mathcal{M}_{0}^{*}$. We are going to apply Step 1 to the projection $\xi_{0}$ of $\xi$ on $\mathcal{M}_{0}^{*}$ and show that the difference $\left|\partial^{2} \operatorname{cap}_{*}\left(B_{1}\right)[\xi, \xi]-\partial^{2} \operatorname{cap}_{*}\left(B_{1}\right)\left[\xi_{0}, \xi_{0}\right]\right|$ is small. Let $\xi$ be in $\mathcal{M}_{\delta}^{*}$. Define

(A)

$$
\xi_{0}:=\xi-\frac{1}{N\left|B_{1}\right|} \int_{\partial B_{1}} \xi d \mathcal{H}^{N-1}
$$

$$
\xi_{0}:=\xi-\frac{1}{N\left|B_{1}\right|} \int_{\partial B_{1}} \xi d \mathcal{H}^{N-1}-\frac{1}{\left|B_{1}\right|} \sum_{i=1}^{N} x_{i} \int_{\partial B_{1}} y_{i} \xi d \mathcal{H}^{N-1} .
$$

It is immediate from the definition that $\xi_{0}$ belongs to $\mathcal{M}_{0}^{*}$. We now compare the norms of $\xi$ and $\xi_{0}$. We denote $c:=\xi-\xi_{0}$ and we write

$$
\begin{aligned}
\left\|\xi_{0}\right\|_{H^{\frac{1}{2}\left(\partial B_{1}\right)}}^{2}= & \int_{\partial B_{1}}(\xi-c)^{2} d \mathcal{H}^{N-1}+\int_{B_{R} \backslash B_{1}}|\nabla H(\xi-c)|^{2} d x \\
= & \|\xi\|_{H^{\frac{1}{2}\left(\partial B_{1}\right)}}^{2}-\|c\|_{H^{\frac{1}{2}\left(\partial B_{1}\right)}}^{2}-2 \int_{\partial B_{1}} c(\xi-c) d \mathcal{H}^{N-1} \\
& -2 \int_{B_{R} \backslash B_{1}} \nabla H(c) \cdot(\nabla H(\xi)-\nabla H(c)) d x \\
= & \|\xi\|_{H^{\frac{1}{2}\left(\partial B_{1}\right)}}^{2}-\|c\|_{H^{\frac{1}{2}}\left(\partial B_{1}\right)}^{2}
\end{aligned}
$$

Note that in the last equality we used integration by parts and the definition of $H$. Since $\xi$ belongs to $\mathcal{M}_{C \delta}^{*}$, we have

$$
\|c\|_{H^{\frac{1}{2}\left(\partial B_{1}\right)}}^{2} \leq C\|c\|_{L^{2}\left(\partial B_{1}\right)}^{2} \leq C \delta^{2}\|\xi\|^{2}
$$

where we have used that since $c=\xi-\xi_{0}$ belongs to an $N+1$ dimensional space, the $H^{1 / 2}$ and the $L^{2}$ are equivalent. Now we apply Step 1 to $\xi_{0}$ to get

$$
\begin{aligned}
\partial^{2} \operatorname{cap}_{*}\left(B_{1}\right)[\xi, \xi] & =\partial^{2} \operatorname{cap}_{*}\left(B_{1}\right)\left[\xi_{0}, \xi_{0}\right]+2 \partial^{2} \operatorname{cap}_{*}\left(B_{1}\right)[\xi, c]-\partial^{2} \operatorname{cap}_{*}\left(B_{1}\right)[c, c] \\
& \geq c\left\|\xi_{0}\right\|_{H^{\frac{1}{2}}\left(\partial B_{1}\right)}^{2}-2\|c\|_{H^{\frac{1}{2}\left(\partial B_{1}\right)}}\|\xi\|_{H^{\frac{1}{2}}\left(\partial B_{1}\right)}-\|c\|_{H^{\frac{1}{2}}\left(\partial B_{1}\right)}^{2}
\end{aligned}
$$

and thus, by (2.3) and (2.4),

$$
\partial^{2} \operatorname{cap}_{*}\left(B_{1}\right)[\xi, \xi] \geq c\left\|\xi_{0}\right\|_{H^{\frac{1}{2}\left(\partial B_{1}\right)}}^{2}-C \delta\|\xi\|^{2} \geq \frac{c}{2}\|\xi\|_{H^{\frac{1}{2}}\left(\partial B_{1}\right)}^{2}
$$

provided $\delta$ is chosen sufficiently small.

\section{Stability FOR BOUnded SETS With SMALl ASYMmetry}

This section is dedicated to the proof of the following theorem.

Theorem 3.1. There exist constants $c=c(N, R), \epsilon_{0}=\epsilon_{0}(N, R)$ such that for any open set $\Omega \subset B_{R}$ with $|\Omega|=\left|B_{1}\right|$ and $\alpha_{*}(\Omega) \leq \epsilon_{0}$ the following inequality holds:

$$
\operatorname{cap}_{*}(\Omega)-\operatorname{cap}_{*}\left(B_{1}\right) \geq c \alpha_{*}(\Omega) \text {. }
$$


We want to reduce our problem to nearly spherical sets. To do that we argue by contradiction. Assume that there exists a sequence of domains $\tilde{\Omega}_{j}$ such that

$$
\left|\tilde{\Omega}_{j}\right|=\left|B_{1}\right|, \alpha_{*}\left(\tilde{\Omega}_{j}\right)=\epsilon_{j} \rightarrow 0, \operatorname{cap}_{*}\left(\tilde{\Omega}_{j}\right)-\operatorname{cap}_{*}\left(B_{1}\right) \leq \sigma^{4} \epsilon_{j}
$$

for some $\sigma$ small enough to be chosen later. We then prove the existence of a new contradicting sequence made of smooth sets via a selection principle.

Theorem 3.2 (Selection Principle). There exists $\tilde{\sigma}=\tilde{\sigma}(N, R)$ such that if one has a contradicting sequence $\tilde{\Omega}_{j}$ as the one described above in (3.1) with $\sigma<\tilde{\sigma}$, then there exists a sequence of smooth open sets $U_{j}$ such that

(i) $\left|U_{j}\right|=\left|B_{1}\right|$,

(ii) $\partial U_{j} \rightarrow \partial B_{1}$ in $C^{k}$ for every $k$,

(iii) $\lim \sup _{j \rightarrow \infty} \frac{\operatorname{cap}_{*}\left(U_{j}\right)-\operatorname{cap}_{*}\left(B_{1}\right)}{\alpha_{*}\left(\Omega_{j}\right)} \leq C \sigma$ for some $C=C(N, R)$ constant,

(iv) for the case of the capacity in $\mathbb{R}^{N}$ the barycenter of every $\Omega_{j}$ is in the origin.

Proof of Theorem 3.1 assuming Selection Principle. Suppose Theorem 3.1 does not hold. Then for any $\sigma>0$ we can find a contradicting sequence $\tilde{\Omega}_{j}$ as in (3.1). We apply Selection Principle to $\tilde{\Omega}_{j}$ to get a smooth contradicting sequence $U_{j}$.

By the properties of $\Omega_{j}$, we have that for $j$ big enough $U_{j}$ is a nearly spherical set. Thus, we can use Theorem 2.8 and get

$$
c(N, R) \leq \limsup _{j \rightarrow \infty} \frac{\operatorname{cap}_{*}\left(U_{j}\right)-\operatorname{cap}_{*}\left(B_{1}\right)}{\alpha_{*}\left(\Omega_{j}\right)} \leq C(N, R) \sigma .
$$

But this cannot happen for $\sigma$ small enough depending only on $N$ and $R$

The proof of Theorem 3.2 is based on constructing the new sequence of sets by solving a variational problem. The existence of this new sequence is established in the next section while its regularity properties are studied in Section 5.

\section{Proof of Theorem 3.2: Existence And First properties}

4.1. Getting rid of the volume constraint. The first step consists in getting rid of the volume constraint in the isocapacitary inequality. Note that this has to be done locally since, by scaling, globally there exists no Lagrange multiplier. Furthermore, to apply the regularity theory for free boundary problems, it is crucial to introduce a monotone dependence on the volume. To this end, let us set

$$
f_{\eta}(s):= \begin{cases}-\frac{1}{\eta}\left(s-\omega_{N}\right), & s \leq \omega_{N} \\ -\eta\left(s-\omega_{N}\right), & s \geq \omega_{N}\end{cases}
$$

and let us consider the new functional

$$
\mathscr{C}_{\eta}^{*}(\Omega)=\operatorname{cap}_{*}(\Omega)+f_{\eta}(|\Omega|) .
$$

We now show that the above functional is uniquely minimized by balls. Note also that $f_{\eta}$ satisfies

$$
\eta(t-s) \leq f_{\eta}(s)-f_{\eta}(t) \leq \frac{(t-s)}{\eta} \quad \text { for all } 0 \leq s \leq t .
$$

Lemma 4.1 (Relative capacity). There exists an $\hat{\eta}=\hat{\eta}(R)>0$ such that the only minimizer of $\mathscr{C}_{\hat{\eta}}^{R}$ in the class of sets contained in $B_{R}$ is $B_{1}$, the unit ball centered at the origin.

Moreover, there exists $c=c(R)>0$ such that for any ball $B_{r}$ with $0<r<R$, one has

$$
\mathscr{C}_{\hat{\eta}}^{R}\left(B_{r}\right)-\mathscr{C}_{\hat{\eta}}^{R}\left(B_{1}\right) \geq c|r-1| .
$$


Lemma 4.2 (Absolute capacity). There exists an $\hat{\eta}=\hat{\eta}(R)>0$ such that the only minimizer of $\mathscr{C}_{\hat{\eta}}$ in the class of sets contained in $B_{R}$ is a translate of the unit unit ball $B_{1}$.

Moreover, there exists $c=c(R)>0$ such that for any ball $B_{r}$ with $0<r<R$, one has

$$
\mathscr{C}_{\hat{\eta}}\left(B_{r}\right)-\mathscr{C}_{\hat{\eta}}\left(B_{1}\right) \geq c|r-1| \text {. }
$$

Proof of Lemma 4.1. First of all, using symmetrization we get that any minimizer of $\mathscr{C}_{\eta}^{R}$ is a ball centered at zero. Thus, it is enough to show that for some $\eta>0$

$$
g(r):=\mathscr{C}_{\eta}^{R}\left(B_{r}\right)
$$

attains its only minimum at $r=1$ on the interval $(0, R)$. We recall that the (relative) capacitary potential of $B_{r}$ in $B_{R}$ is given by

$$
u_{R}=\min \left\{\frac{\left(|x|^{-(n-2)}-R^{-(n-2)}\right)}{r^{-(n-2)}-R^{-(n-2)}}, 1\right\}
$$

and thus

$$
\operatorname{cap}_{R}\left(B_{r}\right)=\frac{(n-2)}{r^{-(n-2)}-R^{-(n-2)}}
$$

hence

$$
g(r)=\operatorname{cap}_{R}\left(B_{r}\right)+f_{\eta}\left(\omega_{N} r^{N}\right)=\frac{R^{N-2}-1}{\left(\frac{R}{r}\right)^{N-2}-1} \operatorname{cap}_{R}\left(B_{1}\right)+f_{\eta}\left(\omega_{N} r^{N}\right) .
$$

For convenience let us denote

and note that

$$
\varphi(r):=\operatorname{cap}_{R}\left(B_{r}\right)=c_{1}(R) \frac{R^{N-2}-1}{R^{N-2}-r^{N-2}} r^{N-2},
$$

$$
\varphi^{\prime}(r)=c_{1}(R)(N-2)\left(\frac{R^{N-2}-1}{R^{N-2}-r^{N-2}} r^{N-3}+\frac{R^{N-2}-1}{\left(R^{N-2}-r^{N-2}\right)^{2}} r^{N-3} r^{N-2}\right) .
$$

Now we consider separately the two cases $0<r \leq 1$ and $1 \leq r \leq R$.

- $0<r \leq 1$

$g^{\prime}(r)=c_{2}(R)\left(\frac{R^{N-2}-1}{R^{N-2}-r^{N-2}} r^{N-3}+\frac{R^{N-2}-1}{\left(R^{N-2}-r^{N-2}\right)^{2}} r^{N-3} r^{N-2}\right)-\frac{1}{\eta} \omega_{N} N r^{N-1}$.

For $r \in(1 / 2,1)$

$$
g^{\prime}(r) \leq c(R)-\frac{1}{\eta} \omega_{N} N\left(\frac{1}{2}\right)^{N-1} .
$$

If we take $\eta<\eta(R) \ll 1$, then $g^{\prime}(r)<-c_{3}(R)$ for $r \in\left(\frac{1}{2}, 1\right)$ and thus $g(r)$ attains its minimum at $r=1$ on that interval.

Moreover for $r \in(0,1 / 2)$

$$
g(r)=\frac{R^{N-2}-1}{\left(\frac{R}{r}\right)^{N-2}-1} \operatorname{cap}_{R}\left(B_{1}\right)+\frac{1}{\eta}\left(\omega_{N}\left(1-r^{N}\right)\right) \geq \frac{1}{\eta}\left(\omega_{N}\left(1-\left(\frac{1}{2}\right)^{N}\right)\right) .
$$

Since $g(1)=\operatorname{cap}_{R}\left(B_{1}\right)=c(R)$ we can take $\eta$ small enough depending only on $R$ to ensure that $g(r) \geq g(1)$ for all $r \in[0,1 / 2)$.

- $1 \leq r<R$

$$
\begin{aligned}
g^{\prime}(r) & =c(R)\left(\frac{R^{N-2}-1}{R^{N-2}-r^{N-2}} r^{N-3}+\frac{R^{N-2}-1}{\left(R^{N-2}-r^{N-2}\right)^{2}} r^{N-3} r^{N-2}\right)-\eta \omega_{N} N r^{N-1} \\
& \geq c(R)-\eta \omega_{N} N R^{N-1} .
\end{aligned}
$$


Taking $\eta \ll 1$ depending only on $r$ we get $g^{\prime}(r)>c_{4}(R)$ for $r \in(1, R)$ and thus $g(r)$ attains its minimum at $r=1$ also on this interval.

To prove the last claim just note that

$$
\lim _{r \rightarrow 1^{-}} g^{\prime}(r) \leq-c_{3} \quad \lim _{r \rightarrow 1^{+}} g^{\prime}(r) \geq c_{4} .
$$

Proof of Lemma 4.2. The proof works exactly as the one in the previous lemma, just using the equality

$$
\operatorname{cap}\left(B_{r}\right)=\operatorname{cap}\left(B_{1}\right) r^{n-2} .
$$

4.2. A penalized minimum problem. The sequence in Theorem 3.2 is obtained by solving the following minimum problem.

$$
\min \left\{\mathscr{C}_{\hat{\eta}, j}^{*}(\Omega): \Omega \subset B_{R}\right\},
$$

where

$\mathscr{C}_{\hat{\eta}, j}^{*}(\Omega)=\mathscr{C}_{\hat{\eta}}^{*}(\Omega)+\sqrt{\epsilon_{j}^{2}+\sigma^{2}\left(\alpha_{*}(\Omega)-\epsilon_{j}\right)^{2}}=\operatorname{cap}_{*}(\Omega)+f_{\hat{\eta}}(|\Omega|)+\sqrt{\epsilon_{j}^{2}+\sigma^{2}\left(\alpha_{*}(\Omega)-\epsilon_{j}\right)^{2}}$.

We start proving the existence of minimizers. As in [BDPV15], in order to ensure the continuity of the asymmetry term, one needs to construct a minimizing sequence with equibounded perimeter. Recall also that a set is said to be quasi open if it is the zero level set of a $W^{1,2}$ function.

Lemma 4.3. There exists $\sigma_{0}=\sigma_{0}(N, R)>0$ such that for every $\sigma<\sigma_{0}$ the minimum in (4.4) is attained by a quasi-open set $\Omega_{j}^{*}$. Moreover, perimeters of $\Omega_{j}^{*}$ are bounded independently on $j$.

Proof. We will focus on the capacity with respect to the ball. For the case of capacity in $\mathbb{R}^{n}$ one simply replaces $W_{0}^{1,2}\left(B_{R}\right)$ by $D^{1,2}\left(\mathbb{R}^{N}\right)$.

Step 1: finding minimizing sequence with bounded perimters. We consider $\left\{V_{k}\right\}_{k \in \mathbb{N}}$ - a minimizing sequence for $\mathscr{C}_{\hat{\eta}, j}^{R}$, satisfying

$$
\mathscr{C}_{\hat{\eta}, j}^{R}\left(V_{k}\right) \leq \inf \mathscr{C}_{\hat{\eta}, j}^{R}+\frac{1}{k}
$$

We denote by $v_{k}$ the capacitary potentials of $V_{k}$, so $V_{k}=\left\{x \in B_{R}: v_{k}=1\right\}$. We take as a variation the slightly enlarged set $\tilde{V}_{k}$ :

$$
\tilde{V}_{k}=\left\{x \in B_{R}: v_{k}>1-t_{k}\right\}
$$

where $t_{k}=\frac{1}{\sqrt{k}}$.

Note that the function $\tilde{v}_{k}=\frac{\min \left(v_{k}, 1-t_{k}\right)}{1-t_{k}}$ is in $W_{0}^{1,2}\left(B_{R}\right)$ and $v_{k}=1$ on $\tilde{V}_{k}$, so we can bound the capacity of $\tilde{V}_{k}$ by $\int_{B_{R}}\left|\nabla \tilde{v}_{k}\right|^{2} d x$. Since $V_{k}$ is almost minimizing, we write

$$
\begin{aligned}
& \int_{\left\{v_{k}<1\right\}}\left|\nabla v_{k}\right|^{2} d x+f_{\hat{\eta}}\left(\left|\left\{v_{k}=1\right\}\right|\right)+\sqrt{\epsilon_{j}^{2}+\sigma^{2}\left(\alpha\left(\left\{v_{k}=1\right\}\right)-\epsilon_{j}\right)^{2}} \\
& \leq \int_{\left\{v_{k}<1-t_{k}\right\}}\left|\nabla\left(\frac{v_{k}}{1-t_{k}}\right)\right|^{2} d x+f_{\hat{\eta}}\left(\left|\left\{v_{k} \geq 1-t_{k}\right\}\right|\right)+\sqrt{\epsilon_{j}^{2}+\sigma^{2}\left(\alpha\left(\left\{v_{k} \geq 1-t_{k}\right\}\right)-\epsilon_{j}\right)^{2}}+\frac{1}{k} .
\end{aligned}
$$


We use (4.1) and the fact that the function $t \mapsto \sqrt{\epsilon_{j}^{2}+\sigma^{2}\left(t-\epsilon_{j}\right)^{2}}$ is 1 Lipschitz to get

$$
\begin{aligned}
& \int_{\left\{1-t_{k}<v_{k}<1\right\}}\left|\nabla v_{k}\right|^{2} d x+\hat{\eta}\left|\left\{1-t_{k}<v_{k}<1\right\}\right| \\
& \leq \sigma\left(\left.\left|\alpha\left(\left\{v_{k} \geq 1-t_{k}\right\}-\alpha\left(\left\{v_{k}=1\right\}\right) \mid\right)+\frac{1}{k}+\int_{\left\{v_{k}<1-t_{k}\right\}}\left(\left(\frac{1}{1-t_{k}}\right)^{2}-1\right)\right| \nabla v_{k}\right|^{2} d x\right. \\
& \leq C(R) \sigma\left|\left\{1-t_{k}<v_{k} \leq 1\right\}\right|+\frac{1}{k}+\left(\left(\frac{1}{1-t_{k}}\right)^{2}-1\right) \operatorname{cap}_{R}\left(V_{k}\right) \\
& \leq C(R) \sigma\left|\left\{1-t_{k}<v_{k} \leq 1\right\}\right|+\frac{1}{k}+c(N, R) t_{k},
\end{aligned}
$$

where in the second inequality we used Lemma 2.3, (ii). Taking $\sigma<\frac{\hat{\eta}}{2 C(R)}$, we obtain

$$
\int_{\left\{1-t_{k}<v_{k}<1\right\}}\left|\nabla v_{k}\right|^{2} d x+\frac{\hat{\eta}}{2}\left(\left|\left\{1-t_{k}<v_{k}<1\right\}\right|\right) \leq \frac{1}{k}+c(N, R) t_{k} .
$$

We estimate the left-hand side from below, using the arithmetic-geometric mean inequality, the Cauchy-Schwarz inequality, and the co-area formula.

$$
\begin{aligned}
& \int_{\left\{1-t_{k}<v_{k}<1\right\}}\left|\nabla v_{k}\right|^{2} d x+\frac{\hat{\eta}}{2}\left(\left|\left\{1-t_{k}<v_{k}<1\right\}\right|\right) \\
& \geq 2\left(\int_{1-t_{k}<v_{k}<1}\left|\nabla v_{k}\right|^{2} d x\right)^{\frac{1}{2}}\left(\frac{\hat{\eta}}{2}\left(\left|\left\{1-t_{k}<v_{k}<1\right\}\right|\right)\right)^{\frac{1}{2}} \\
& \geq \sqrt{2 \hat{\eta}} \int_{1-t_{k}<v_{k}<1}\left|\nabla v_{k}\right| d x=\sqrt{2 \hat{\eta}} \int_{1-t_{k}}^{1} P\left(v_{k}>s\right) d s .
\end{aligned}
$$

where $P(E)$ denotes the De Giorgi perimeter of a set $E$. Hence, there exists a level $1-t_{k}<s_{k}<1$ such that for $\hat{V}_{k}=\left\{v_{k}>s_{k}\right\}$

$$
P\left(\hat{V}_{k}\right) \leq \frac{1}{t_{k}} \int_{1-t_{k}}^{1} P\left(\left\{v_{k}>s\right\}\right) d s \leq \frac{1}{t_{k} \sqrt{2 \hat{\eta} k}}+c(N, R)=\frac{1}{\sqrt{2 \hat{\eta} k}}+c(N, R) .
$$

where in the last equality we have used that $t_{k}=\frac{1}{\sqrt{k}}$. These $\hat{V}_{k}$ will give us the desired "good" minimizing sequence, indeed

$$
\begin{aligned}
& \mathscr{C}_{\hat{\eta}, j}^{R}\left(\hat{V}_{k}\right) \\
& \quad \leq \mathscr{C}_{\hat{\eta}, j}^{R}\left(V_{k}\right)+f_{\hat{\eta}}\left(\left|\left\{v_{k}>s_{k}\right\}\right|\right)-f_{\hat{\eta}}\left(\left|\left\{v_{k}=1\right\}\right|\right)+C \sigma\left|\left\{1-s_{k}<v_{k}<1\right\}\right| \leq \mathscr{C}_{\hat{\eta}, j}^{R}\left(V_{k}\right),
\end{aligned}
$$

where in the first inequality we have used that $\hat{V}_{k} \subset V_{k}$ and in the second that, thanks to our choice of $\sigma$,

$f_{\hat{\eta}}\left(\left|\left\{v_{k}>s_{k}\right\}\right|\right)-f_{\hat{\eta}}\left(\left|\left\{v_{k}=1\right\}\right|\right)+C \sigma\left|\left\{1-s_{k}<v_{k}<1\right\} \leq(C \sigma-\hat{\eta})\right|\left\{1-s_{k}<v_{k}<1\right\} \leq 0$.

Step 2: Existence of a minimizer. Since $\left\{\hat{V}_{k}\right\}_{k}$ is a sequence with equibounded perimeter,s there exists a Borel set $\hat{V}_{\infty}$ such that up to a (not relabelled) subsequence

$$
1_{\hat{V}_{k}} \rightarrow 1_{\hat{V}_{\infty}} \text { in } L_{1}\left(B_{R}\right) \text { and a.e. in } B_{R}, \quad P\left(\hat{V}_{\infty}\right) \leq C(N, R) \text {. }
$$

We want to show that $\hat{V}_{\infty}$ is a minimizer for $\mathcal{C}_{\eta, j}$. We set $\hat{v}_{k}=\frac{\min \left(v_{k}, s_{k}\right)}{s_{k}}$ and we note that they are the capacitary potentials of $\hat{V}_{k}$. Moreover the sequence $\left\{\hat{v}_{k}\right\}_{k}$ is bounded in 
$W_{0}^{1,2}\left(B_{R}\right)$. Thus, there exists a function $\hat{v} \in W_{0}^{1,2}\left(B_{R}\right)$ such that up to a (not relabelled) subsequence

$$
\hat{v}_{k} \rightarrow \hat{v} \text { strongly in } L^{2}\left(B_{R}\right) \text { and a.e. in } B_{R} \text {. }
$$

Let us define $\hat{V}=\{x: \hat{v}=1\}$, we want to show that $\hat{V}$ is a minimizer. First, note that

$$
1_{\hat{V}}(x) \geq \limsup 1_{\hat{V}_{k}}(x)=1_{\hat{V}_{\infty}}(x) \quad \text { for a.e. } x \in B_{R},
$$

hence $\left|\hat{V}_{\infty} \backslash \hat{V}\right|=0$. Moreover, by the lower semicontinuity of Dirichlet integral, the monotonicity of $f_{\hat{\eta}}$ and the continuity of $\alpha$ with respect to the $L^{1}$ convergence, we have

$$
\begin{aligned}
\inf \mathscr{C}_{\hat{\eta}, j}^{R} & =\lim _{k} \int\left|\nabla \hat{v}_{k}\right|^{2}+f_{\hat{\eta}}\left(\left|\hat{V}_{k}\right|\right)+\sqrt{\epsilon_{j}^{2}+\sigma^{2}\left(\alpha\left(\hat{V}_{k}\right)-\epsilon_{j}\right)^{2}} \\
& \geq \operatorname{cap}_{R}(\hat{V})+f_{\hat{\eta}}\left(\left|\hat{V}_{\infty}\right|\right)+\sqrt{\epsilon_{j}^{2}+\sigma^{2}\left(\alpha\left(\hat{V}_{\infty}\right)-\epsilon_{j}\right)^{2}} . \geq \operatorname{cap}_{R}(\hat{V})+f_{\hat{\eta}}(|\hat{V}|)
\end{aligned}
$$

Hence

$$
\begin{aligned}
\operatorname{cap}_{R}(\hat{V}) & +f_{\hat{\eta}}\left(\left|\hat{V}_{\infty}\right|\right)+\sqrt{\epsilon_{j}^{2}+\sigma^{2}\left(\alpha\left(\hat{V}_{\infty}\right)-\epsilon_{j}\right)^{2}} \leq \inf \mathscr{C}_{\hat{\eta}, j}^{R}(\Omega) \\
& \leq \operatorname{cap}_{R}(\hat{V})+f_{\hat{\eta}}(|\hat{V}|)+\sqrt{\epsilon_{j}^{2}+\sigma^{2}\left(\alpha(\hat{V})-\epsilon_{j}\right)^{2}} .
\end{aligned}
$$

Using Lemma 2.3 (ii) we get

$$
f_{\hat{\eta}}\left(\left|\hat{V}_{\infty}\right|\right)-f_{\hat{\eta}}(|\hat{V}|) \leq C \sigma\left|\hat{V} \Delta \hat{V}_{\infty}\right|=C \sigma\left|\hat{V} \backslash \hat{V}_{\infty}\right| .
$$

Since $|\hat{V}| \geq\left|\hat{V}_{\infty}\right|,(4.1)$ and our choice of $\sigma$ yield

$$
\hat{\eta}\left|\hat{V} \backslash \hat{V}_{\infty}\right| \leq f_{\hat{\eta}}\left(\left|\hat{V}_{\infty}\right|\right)-f_{\hat{\eta}}(|\hat{V}|) \leq C \sigma\left|\hat{V} \backslash \hat{V}_{\infty}\right| \leq \frac{\hat{\eta}}{2}\left|\hat{V} \backslash \hat{V}_{\infty}\right|
$$

from which we conclude that $\left|\hat{V} \Delta \hat{V}_{\infty}\right|=0$ and thus, by (4.5) that $\hat{V}$ is the desired minimizer.

4.3. First properties of the minimizers. Let us conclude by establishing some properties of the minimizers of (4.4).

Lemma 4.4. Let $\left\{\Omega_{j}\right\}$ be a sequence of minimizers for (4.4). Then the following properties hold:

(i) $\left|\alpha_{*}\left(\Omega_{j}\right)-\epsilon_{j}\right| \leq 3 \sigma \epsilon_{j}$;

(ii) ||$\Omega_{j}|-| B_{1}|| \leq C \sigma^{4} \epsilon_{j}$;

(iii) (A) for the capacity in $\mathbb{R}^{n}$ up to translations $\Omega_{j} \rightarrow B_{1}$ in $L^{1}$,

(B) for the relative capacity $\Omega_{j} \rightarrow B_{1}$ in $L^{1}$;

(iv) $0 \leq \mathscr{C}_{\hat{\eta}}^{*}\left(\Omega_{j}\right)-\mathscr{C}_{\hat{\eta}}^{*}\left(B_{1}\right) \leq \sigma^{4} \epsilon_{j}$.

Proof. Recall that the sequence $\left\{\Omega_{j}\right\}$ was obtained by a sequence $\left\{\tilde{\Omega}_{j}\right\}$ satisying

(1) $\left|\tilde{\Omega}_{j}\right|=\left|B_{1}\right|$,

(2) $\alpha_{*}\left(\tilde{\Omega}_{j}\right)=\epsilon_{j}$,

(3) $\operatorname{cap}_{*}\left(\tilde{\Omega}_{j}\right)-\operatorname{cap}_{*}\left(B_{1}\right) \leq \sigma^{4} \epsilon_{j}$.

We now use $\left\{\tilde{\Omega}_{j}\right\}$ as comparison domains for the functionals $\mathscr{C}_{\hat{\eta}, j}^{*}$ to get

$$
\mathscr{C}_{\hat{\eta}}^{*}\left(\Omega_{j}\right)+\epsilon_{j} \leq \mathscr{C}_{\hat{\eta}, j}^{*}\left(\Omega_{j}\right) \leq \mathscr{C}_{\hat{\eta}, j}^{*}\left(\tilde{\Omega}_{j}\right)=\mathscr{C}_{\hat{\eta}}^{*}\left(\tilde{\Omega}_{j}\right)+\epsilon_{j} \leq \mathscr{C}_{\hat{\eta}}^{*}\left(B_{1}\right)+\epsilon_{j}\left(1+\sigma^{4}\right),
$$

implying that

$$
\mathscr{C}_{\hat{\eta}}^{*}\left(\Omega_{j}\right)-\mathscr{C}_{\hat{\eta}}^{*}\left(B_{1}\right) \leq \epsilon_{j} \sigma^{4}
$$


which proves (iv). Note that we defined $f_{\hat{\eta}}$ in such a way that $\mathscr{C}_{\hat{\eta}}^{*}\left(\Omega_{j}\right) \geq \mathscr{C}_{\hat{\eta}}^{*}\left(B_{1}\right)$. Thus, using (4.6) we also deduce that

$$
\sqrt{\epsilon_{j}^{2}+\sigma^{2}\left(\alpha_{*}\left(\Omega_{j}\right)-\epsilon_{j}\right)^{2}} \leq \epsilon_{j}\left(1+\sigma^{4}\right)
$$

which gives (i). To estimate the volume of $\Omega_{j}$, we use the classical isocapacitary inequality and properties of $f_{\hat{\eta}}$ and (4.2), (4.3). Indeed, let $B^{j}$ be the ball centered in the origin such that $\left|B^{j}\right|=\left|\Omega_{j}\right|$.Then

$$
\sigma^{4} \epsilon_{j} \geq \mathscr{C}_{\hat{\eta}}^{*}\left(\Omega_{j}\right)-\mathscr{C}_{\hat{\eta}}^{*}\left(B_{1}\right) \geq \mathscr{C}_{\hat{\eta}}^{*}\left(B^{j}\right)-\mathscr{C}_{\hat{\eta}}^{*}\left(B_{1}\right) \geq c(R)|| \Omega_{j}|-| B_{1}||,
$$

where in the last inequality we have used (4.2), (4.3). This proves (ii). To prove (ii) we recall that the sets $\Omega_{j}$ have equibounded perimeter. Hence, the sequence $\left\{\Omega_{j}\right\}_{j}$ is precompact in $L^{1}\left(B_{R}\right)$. Since the asymmetry is continuous with respect to $L^{1}$ convergence any limit set has zero asymmetry. The only set with zero asymmetry is the unit ball (or a translated unit ball in the case of the absolute), proving (iii).

\section{Proof of Theorem 3.2: Regularity}

In this section, we show that the sequence of minimizers of (3) converges smoothly to the unit ball. This will be done by relying on the regularity theory for free boundary problems established in [AC81].

5.1. Linear growth away from the free boundary. Let $u_{j}$ be the capacitary potential for $\Omega_{j}$, a minimizer of (4.4). Let us $v_{j}:=1-u_{j}$, so that $\Omega_{j}=\left\{v_{j}=0\right\}, v_{j}=1$ on $\partial B_{R}$, following [AC81] we are going to show that

$$
v_{j}(x) \sim \operatorname{dist}\left(x, \Omega_{j}\right) .
$$

where the implicit constant depends only on $R$. The above estimate is obtained by suitable comparison estimates. In order to be able to perform them with constants which depend only on $R$, we need to know that $\left\{u_{j}=1\right\}$ is uniformly far from $\partial B_{R}$. This will be achieved by first establishing (uniform in $j$ ) Hölder continuity of $u_{j}$.

5.1.1. Hölder continuity. The proof of Hölder continuity is quite standard and it is based on establishing a decay estimate for the integral oscillation of $u_{j}$. Since, thanks to the minimizing property, $u_{j}$ is close to the harmonic function in $B_{r}\left(x_{0}\right) \cap B_{R}$ with the same boundary value, we start by recalling the decay of the harmonic functions both in the interior and at the boundary. The following is well known, see for instance [GM12, Proposition $5.8]$.

Lemma 5.1. Suppose $w \in W^{1,2}(\Omega)$ is harmonic, $x_{0} \in \Omega$. Then there exists a constant $c=c(n)$ such that for any balls $B_{r_{1}}\left(x_{0}\right) \subset B_{r_{2}}\left(x_{0}\right) \Subset \Omega$

$$
f_{B_{r_{1}}\left(x_{0}\right)}\left(w-f_{B_{r_{1}}\left(x_{0}\right)} w\right)^{2} \leq c\left(\frac{r_{1}}{r_{2}}\right)^{2} f_{B_{r_{2}}\left(x_{0}\right)}\left(w-f_{B_{r_{2}}\left(x_{0}\right)} w\right)^{2} .
$$

Next lemma studies the decay at the boundary, the result is well known. Since we have not been able to find a precise reference for this statement, we report its simple proof.

Lemma 5.2. Let $\Omega$ be an open set such that $0 \in \partial \Omega$ and let $w \in W^{1,2}\left(B_{r}\right)$ be harmonic in $\Omega \cap B_{r}, w \equiv 0$ on $B_{r} \backslash \Omega$. Assume that there exists $\delta>0$ such that for $\rho \leq r$

$$
\frac{\left|\Omega^{c} \cap B_{\rho}\right|}{\left|B_{\rho}\right|} \geq \delta
$$


Then there exist a constant $c=c(\delta)$ and an exponent $\gamma=\gamma(\delta)>0$ such that for any $0<r_{1}<r_{2}<r$ we have

$$
f_{B_{r_{1}}} w^{2} \leq c\left(\frac{r_{1}}{r_{2}}\right)^{\gamma} f_{B_{r_{2}}} w^{2}
$$

Remark 5.3. Note that as $w$ is harmonic in $\Omega \cap B_{r}$ and 0 on $B_{r} \backslash \Omega, w^{2}$ is subharmonic in $B_{r}$, thus its means over balls increase with the radius. In particular,

$$
\sup _{B_{r}} w^{2} \leq c(N) f_{B_{3 r}} w^{2} .
$$

Proof of Lemma 5.2. For convenience, we assume that $r>1$ (we can reduce to this case by scaling). First, we note that it is enough to show the result for radii with the ratio equal to a power of $\frac{1}{4}$. Indeed, take $k \in \mathbb{Z}_{+}$such that $\frac{1}{4^{k+1}} \leq \frac{r_{1}}{r_{2}}<\frac{1}{4^{k}}$. Then

$$
f_{B_{r_{1}}} w^{2} \leq C 4^{-\gamma k} f_{B_{r_{1} 4^{k}}} w^{2} \leq C 4^{-\gamma k} f_{B_{r_{2}}} w^{2} \leq C 4^{\gamma}\left(\frac{r_{1}}{r_{2}}\right)^{-\gamma} f_{B_{r_{2}}} w^{2} .
$$

We work with powers of $\frac{1}{4}$. We start by showing

$$
\sup _{B_{\frac{1}{4}}} w \leq(1-c) \sup _{B_{1}} w
$$

For any $\epsilon>0$ there exists some $x_{0} \in B_{\frac{1}{4}}$ such that $\sup _{B_{\frac{1}{4}}} w \leq w\left(x_{0}\right)+\epsilon$, so we can write

$$
\begin{aligned}
& \sup _{B_{\frac{1}{4}}} w-\epsilon \leq w\left(x_{0}\right) \leq f_{B_{\frac{3}{4}}\left(x_{0}\right)} w \leq \frac{\left|\Omega \cap B_{\frac{3}{4}}\left(x_{0}\right)\right|}{\left|B_{\frac{3}{4}}\left(x_{0}\right)\right|} \sup _{B_{1}} w \\
& =\left(1-\frac{\left|\Omega^{c} \cap B_{\frac{3}{4}}\left(x_{0}\right)\right|}{\left|B_{\frac{3}{4}}\left(x_{0}\right)\right|}\right) \sup _{B_{1}} w \leq\left(1-\frac{\left|\Omega^{c} \cap B_{\frac{1}{4}}\right|}{\left|B_{\frac{3}{4}}\right|}\right) \sup _{B_{1}} w \\
& \leq\left(1-\delta \frac{\left|B_{\frac{1}{4}}\right|}{\left|B_{\frac{3}{4}}\right|}\right) \sup _{B_{1}} w,
\end{aligned}
$$

which proves (5.3) since $\epsilon$ is arbitrary. Using induction and scaling we can extend this result to all powers of $\frac{1}{4}$. Indeed $\tilde{w}(x)=w(x / 4)$ satisfies the hypothesis of the theorem. Hence,

and thus

$$
\sup _{B_{\frac{1}{16}}} w=\sup _{B_{\frac{1}{4}}} \tilde{w} \leq(1-c) \sup _{B_{1}} \tilde{w}=(1-c) \sup _{B_{\frac{1}{4}}} w \leq(1-c)^{2} \sup _{B_{1}} w
$$

In the same way

$$
\sup _{B_{\frac{1}{4^{k}}}} w \leq(1-c)^{k} \sup _{B_{1}} w
$$

$$
\sup _{B_{\frac{1}{4^{k}}}} w \leq(1-c)^{k} \sup _{B_{r}} w
$$

Now

$$
\begin{aligned}
f_{B_{\frac{1}{4^{k}}}} w^{2} & \leq\left(\sup _{B_{\frac{1}{4^{k}}}} w\right)^{2} \leq(1-c)^{2(k-1)}\left(\sup _{B_{\frac{1}{4}}} w\right)^{2} \\
& \leq(1-c)^{2(k-1)}\left(f_{B_{\frac{3}{4}}\left(x_{0}\right)} w^{2}\right) \leq(1-c)^{2(k-1)}\left(c^{\prime} f_{B_{1}} w^{2}\right),
\end{aligned}
$$


where we have used (5.2). We get from powers of $\frac{1}{4}$ to other radii again by scaling. This concludes the proof with $\gamma=-\log _{4}(1-c)$.

Corollary 5.4. Let $w$ as in the statement of Lemma 5.2, then

$$
f_{B_{r_{1}}}\left(w-f_{B_{r_{1}}} w\right)^{2} \leq C\left(\frac{r_{1}}{r_{2}}\right)^{\gamma} f_{B_{r_{2}}}\left(w-f_{B_{r_{2}}} w\right)^{2}
$$

for any $0<r_{1}<r_{2}<r$ with $C$ a constant depending only on $\delta$.

Proof. The proof follows from Lemma 5.2 and the simple observation that for a function $w$ vanishing on a fixed fraction of $B_{\rho}$, the $L^{2}$ norm and the variance are comparable. Namely there exists a constant $c=c(\delta)$ such that

$$
\frac{1}{c} \int_{B_{\rho}}\left(w-f_{B_{\rho}} w\right)^{2} \leq \int_{B_{\rho}} w^{2} \leq c \int_{B_{\rho}}\left(w-f_{B_{\rho}} w\right)^{2} .
$$

Indeed, the first inequality is true for every $w$ with $c=1$. For the second one note that

$$
\int_{B_{\rho}}\left(w-f_{B_{\rho}} w\right)^{2}=\int_{B_{\rho}} w^{2}-\left|B_{\rho}\right|\left(f_{B_{\rho}} w\right)^{2} .
$$

Hence we need to estimate $\left(f_{B_{\rho}} w\right)^{2}$ in terms of $\int_{B_{\rho}} w^{2}$. Since $w$ is non-zero only inside $\Omega$, using Hölder inequality, we obtain

$$
\left(f_{B_{\rho}} w\right)^{2} \leq\left(\frac{\left|\Omega \cap B_{\rho}\right|}{\left|B_{\rho}\right|}\right) f_{B_{\rho}} w^{2} \leq(1-\delta) f_{B_{\rho}} w^{2},
$$

hence

$$
\int_{B_{\rho}}\left(w-f_{B_{\rho}} w\right)^{2} \geq \delta \int_{B_{\rho}} w^{2}
$$

concluding the proof.

To prove Hölder continuity of $u_{j}$ we will use several times the following comparison estimates.

Lemma 5.5. Let $u_{j}$ be the capacitary potential of a minimizer for (4.4). Let $A \subset B_{R}$ be an open set with Lipschitz boundary and let $w \in W^{1,2}\left(\mathbb{R}^{n}\right)$ coincide with $u_{j}$ on the boundary of $A$ in the sense of traces.

Then

$$
\int_{A}\left|\nabla u_{j}\right|^{2} d x-\int_{A}|\nabla w|^{2} d x \leq\left(\frac{1}{\hat{\eta}}+C \sigma\right)|A \cap(\{u=1\} \Delta\{w=1\})| .
$$

Moreover, if $u_{j} \leq w \leq 1$ in $A$, then

$$
\int_{A}\left|\nabla u_{j}\right|^{2} d x+\frac{\hat{\eta}}{2}|A \cap(\{u=1\} \Delta\{w=1\})| \leq \int_{A}|\nabla w|^{2} d x,
$$

provided $\sigma \leq \sigma(R)$.

Proof. We prove the result for the relative capacity. The case of the capacity in $\mathbb{R}^{N}$ can be treated in the same way. Since $u_{j}$ is fixed we drop the subscript $j$. Consider $\tilde{u}$ defined as

$$
\left\{\begin{array}{ll}
\tilde{u}=w & \text { in } A \\
\tilde{u}=u & \text { else. }
\end{array} .\right.
$$


Take $\tilde{\Omega}=\{\tilde{u}=1\}$ as a comparison domain. Since $\Omega$ is minimizing, we can write

$$
\begin{aligned}
& \int_{B_{R}}|\nabla u|^{2} d x+f_{\hat{\eta}}(\Omega)+\sqrt{\epsilon_{j}^{2}+\sigma^{2}\left(\alpha_{R}(\Omega)-\epsilon_{j}\right)^{2}}=\mathscr{C}_{\hat{\eta}, j}^{R}(\Omega) \\
& \leq \mathscr{C}_{\hat{\eta}, j}^{R}(\tilde{\Omega}) \leq \int_{B_{R}}|\nabla \tilde{u}|^{2} d x+f_{\hat{\eta}}(\tilde{\Omega})+\sqrt{\epsilon_{j}^{2}+\sigma^{2}\left(\alpha(\tilde{\Omega})-\epsilon_{j}\right)^{2}} .
\end{aligned}
$$

Hence, by Lemma 2.3, (ii) and (4.1).

$$
\int_{A}|\nabla u|^{2} d x-\int_{A}|\nabla w|^{2} d x \leq\left|f_{\hat{\eta}}(\Omega)-f_{\hat{\eta}}(\tilde{\Omega})\right|+C \sigma|\Omega \Delta \tilde{\Omega}| \leq\left(\frac{1}{\hat{\eta}}+C \sigma\right)|\Omega \Delta \tilde{\Omega}| .
$$

To prove the second inequality we observe that $u \leq w \leq 1$ implies $\{u=1\} \subset\{\tilde{u}=1\}$, i.e. $\Omega \subset \tilde{\Omega}$. Hence, by (4.1):

$$
\int_{A}|\nabla u|^{2} d x-\int_{A}|\nabla w|^{2} d x \leq-f_{\hat{\eta}}(\Omega)+f_{\hat{\eta}}(\tilde{\Omega})+C \sigma|\Omega \Delta \tilde{\Omega}| \leq-\hat{\eta}|\tilde{\Omega} \backslash \Omega|+C \sigma|\tilde{\Omega} \backslash \Omega|,
$$

from which the inequality follows choosing $\sigma$ small enough.

Remark 5.6. Note that if $w$ is harmonic in $A$, then

$$
\int_{A}|\nabla u|^{2} d x-\int_{A}|\nabla w|^{2} d x=\int_{A}|\nabla(u-w)|^{2} d x,
$$

meaning that the first inequality from the lemma becomes

$$
\int_{A}|\nabla(u-w)|^{2} d x \leq\left(\frac{1}{\hat{\eta}}+C \sigma\right)|A \cap\{u=1\} \Delta\{w=1\}| .
$$

Let us also recall the following technical result

Lemma 5.7 ( [Lemma 5.13 in [GM12]). Let $\phi: \mathbb{R}^{+} \rightarrow \mathbb{R}^{+}$be a non-decreasing function satisfying

$$
\phi(\rho) \leq A\left[\left(\frac{\rho}{R}\right)^{\alpha}+\epsilon\right] \phi(R)+B R^{\beta},
$$

for some $A, \alpha, \beta>0$, with $\alpha>\beta$ and for all $0<\rho \leq R \leq R_{0}$, where $R_{0}>0$ is given. Then there exist constants $\epsilon_{0}=\epsilon_{0}(A, \alpha, \beta)$ and $c=c(A, \alpha, \beta)$ such that if $\epsilon \leq \epsilon_{0}$, we have

$$
\phi(\rho) \leq c\left[\frac{\phi(R)}{R^{\beta}}+B\right] \rho^{\beta}
$$

for all $0 \leq \rho \leq R \leq R_{0}$.

Lemma 5.8. There exists $\alpha \in(0,1 / 2)$ such that every minimizer of (4.4) staisfies $u_{j} \in$ $C^{0, \alpha}\left(\overline{B_{R}}\right)$. Moreover, the Hölder norm is bounded by a constant independent on $j$.

Proof. Let us extend $u_{j}$ by 0 outside of $B_{R}$. As usual, we drop the subscript $j$. By Camapanato's criterion it is enough to show that

$$
\phi(r):=\int_{B_{r}\left(x_{0}\right)}\left|u-f_{B_{r}\left(x_{0}\right)} u\right|^{2} \leq C r^{2 \alpha}
$$

for all $r$ small enough (say less that $1 / 2$ ).

Step 1: estimates on the boundary. Let $x_{0} \in \partial B_{R}$. Let $w$ be the harmonic extension of $u$ in $B_{r^{\prime}}\left(x_{0}\right) \cap B_{R}$. By Corollary 5.4 we know that

$$
\int_{B_{r}\left(x_{0}\right)}\left|w-f_{B_{r}\left(x_{0}\right)} w\right|^{2} \leq C\left(\frac{r}{r^{\prime}}\right)^{N+\gamma} \int_{B_{r^{\prime}}\left(x_{0}\right)}\left|w-f_{B_{r^{\prime}}\left(x_{0}\right)} w\right|^{2}
$$


for some $\gamma>0$. Let $g:=u-w$. Then

$$
\begin{aligned}
\int_{B_{r}\left(x_{0}\right)}\left|u-f_{B_{r}\left(x_{0}\right)} u\right|^{2} d x & \leq 2 \int_{B_{r}\left(x_{0}\right)}\left|w(x)-f_{B_{r}\left(x_{0}\right)} w\right|^{2} d x+2 \int_{B_{r}\left(x_{0}\right)}\left|g-f_{B_{r}\left(x_{0}\right)} g\right|^{2} \\
& \leq 2 C\left(\frac{r}{r^{\prime}}\right)^{N+\gamma} \int_{B_{r^{\prime}}\left(x_{0}\right)}\left|w-f_{B_{r}\left(x_{0}\right)} w\right|^{2} d x+2 \int_{B_{r^{\prime}}\left(x_{0}\right)}|g|^{2} \\
& \leq C\left(\frac{r}{r^{\prime}}\right)^{N+\gamma} \int_{B_{r^{\prime}}\left(x_{0}\right)}\left|u-f_{B_{r}\left(x_{0}\right)} u\right|^{2} d x+C \int_{B_{r^{\prime}}\left(x_{0}\right)}|g|^{2}
\end{aligned}
$$

To estimate $\int_{B_{r^{\prime}}\left(x_{0}\right)}|g(x)|^{2} d x$ we recall that $g \in W_{0}^{1,2}\left(B_{r^{\prime}}\left(x_{0}\right)\right)$ and vanishes outside $B_{r^{\prime}}\left(x_{0}\right) \cap B_{R}$, hence by Poincaré's inequality and (5.4)

$$
\int_{B_{r^{\prime}}\left(x_{0}\right)}|g|^{2} \leq C\left(r^{\prime}\right)^{2} \int_{B_{r^{\prime}}\left(x_{0}\right) \cap B_{R}}|\nabla g|^{2} \leq C\left(r^{\prime}\right)^{N+2}
$$

Combining the last two inequalities, we get

$$
\phi(r) \leq c\left(\frac{r}{r^{\prime}}\right)^{N+\gamma} \phi\left(r^{\prime}\right)+C\left(r^{\prime}\right)^{N+2} .
$$

Using Lemma 5.7 we obtain

$$
\phi(r) \leq c\left(\left(\frac{r}{r^{\prime}}\right)^{N+\gamma} \phi\left(r^{\prime}\right)+C r^{N+\gamma}\right)
$$

for any $r<r^{\prime}<1$. In particular,

$$
\phi(r) \leq c\left(\|u\|_{L^{2}\left(\mathbb{R}^{N}\right)}^{2}+C\right) r^{N+\gamma} .
$$

Step 2: estimates at the interior. Assume that $x_{0} \in B_{R}, r<r^{\prime}<\operatorname{dist}\left(x_{0}, \partial B_{R}\right)$, so that $B_{r}\left(x_{0}\right) \subset B_{r^{\prime}}\left(x_{0}\right) \subset B_{R}$. Then one can proceed in the same way as in the previous step using Lemma 5.1 instead of Corollary 5.4. Hence

$$
\phi(r) \leq C\left(\left(\frac{r}{r^{\prime}}\right)^{N+\gamma} \phi\left(r^{\prime}\right)+C r^{N+\gamma}\right)
$$

for $r<r^{\prime}<\operatorname{dist}\left(x_{0}, \partial B_{R}\right)$ and, in particular,

$$
\phi(r) \leq c\left(\left(\frac{1}{\operatorname{dist}\left(x_{0}, \partial B_{R}\right)}\right)^{N+\gamma}\|u\|_{L^{2}\left(\mathbb{R}^{N}\right)}^{2}+C\right) r^{N+\gamma} .
$$

Step 3: global estimates. We now combine the previous steps, distinguishing several cases:

- $\operatorname{dist}\left(x_{0}, \partial B_{R}\right)>1 / 2$. By Step 2

$$
\phi(r) \leq C\left(\|u\|_{L^{2}\left(\mathbb{R}^{N}\right)}^{2}+C\right) r^{N+\gamma} .
$$


- $r \leq \rho:=\operatorname{dist}\left(x_{0}, \partial B_{R}\right) \leq 1 / 2$. Let $y_{0}=R \frac{x_{0}}{\left|x_{0}\right|}$ be the intersection of the ray $\left[0, x_{0}\right)$ with $\partial B_{R}$. Then, using Step 2 and Step 1, we have

$$
\begin{aligned}
\phi(r) & \leq C\left(\left(\frac{r}{\rho}\right)^{N+\gamma} \phi(\rho)+C r^{N+\gamma}\right) \\
& =C\left(\left(\frac{r}{\rho}\right)^{N+\gamma} \int_{B_{\rho}\left(x_{0}\right)}\left|u-f_{B_{\rho}\left(x_{0}\right)} u\right|^{2}+C r^{N+\gamma}\right) \\
& \leq C\left(\left(\frac{r}{\rho}\right)^{N+\gamma} \int_{B_{2 \rho}\left(y_{0}\right)}\left|u-f_{B_{2 \rho}\left(y_{0}\right)} u\right|^{2}+C r^{N+\gamma}\right) \\
& \leq C\left(\left(\frac{r}{\rho}\right)^{N+\gamma}(2 \rho)^{N+\gamma} \int_{B_{1}\left(y_{0}\right)}\left|u-f_{B_{1}\left(y_{0}\right)} u\right|^{2}+C r^{N+\gamma}\right) \\
& \leq C\left(\|u\|_{L^{2}\left(\mathbb{R}^{N}\right)}^{2}+C\right) r^{N+\gamma} .
\end{aligned}
$$

- $\rho:=\operatorname{dist}\left(x_{0}, \partial B_{R}\right) \leq r \leq 1 / 2$. Again we set $y_{0}$ to be the radial projection of $x_{0}$ onto $\partial B_{R}$. We use Step 1 and get

$$
\begin{aligned}
\phi(r) & =\int_{B_{r}\left(x_{0}\right)}\left|u-f_{B_{r}\left(x_{0}\right)} u\right|^{2} \leq \int_{B_{2 r}\left(y_{0}\right)}\left|u-f_{B_{2 r}\left(y_{0}\right)} u\right|^{2} \\
& \leq C\left(r^{N+\gamma} \int_{B_{1}\left(y_{0}\right)}\left|u-f_{B_{1}\left(y_{0}\right)} u\right|^{2}+C r^{N+\gamma}\right) \\
& \leq C\left(\|u\|_{L^{2}\left(\mathbb{R}^{N}\right)}^{2}+C\right) r^{N+\gamma} .
\end{aligned}
$$

In conclusion,

$$
\phi(r) \leq C\left(\|u\|_{L^{2}\left(\mathbb{R}^{N}\right)}^{2}+C\right) r^{N+\gamma},
$$

which by Campanato criterion implies that $u \in C^{\frac{\gamma}{2}}$. Note furthermore that the dependence on $j$ is realized only by the $L^{2}$ norm of $u_{j}$ which is uniformly bounded by $\sqrt{\left|B_{R}\right|}$.

5.1.2. Lipschitz continuity and density estimates on the boundary. We now prove two lemmas similar to those in Section 3 of [AC81]. These are obtained by adding or removing a small ball from an optimizer of (4.4). Since our competitors are constrained to lie in $B_{R}$ removing a ball is not a problem. On the other hand adding might lead to a non admissible competitor. For the case of the relative capacity, we use the Hölder estimate of the previous section. Indeed it implies that there exists $\rho_{0}=\rho_{0}(R)>0$ such that

$$
\Omega_{j} \subset B_{R-\rho_{0}} .
$$

Lemma 5.9. For $\kappa<1$ there is a constant $c=c(N, \kappa, R)$ such that if $u_{j}$ is a minimizer for (4.4) and $v_{j}=1-u_{j}$ satisfies

$$
f_{\partial B_{r}\left(x_{0}\right)} v_{j} \leq c r
$$

then $v_{j}=0$ in $B_{\kappa r}\left(x_{0}\right)$. In the case of the relative capacity we assume $r \leq \rho_{0}$ where $\rho_{0}$ is as in (5.6). 
Proof. We drop the subscript $j$ for simplicity. We first check that $B_{\kappa r}\left(x_{0}\right) \subset B_{R}$. By our restriction on $r$ this is clear in the case of the relative capacity. Let us show that this is the case also for the absolute capacity provided we choose $c$ small enough (depending only on $R$ and $N, \kappa)$. To prove this we use that $v$ cannot be too small outside of $B_{R}$. More precisely, by comparison principle we know that

$$
v(x) \geq v_{B_{R}}(x)=1-\frac{R^{N-2}}{|x|^{N-2}},
$$

where $v_{B_{R}}$ is the corresponding function for $B_{R}$. Suppose that $B_{\kappa r}\left(x_{0}\right) \backslash B_{R} \neq \emptyset$. Then the part of $\partial B_{r}\left(x_{0}\right) \backslash B_{R}$ with the distance at least $\frac{1-\kappa}{2} r$ from the boundary of the ball $B_{R}$ has measure at least $c(\kappa) r^{N-1}$. Then

$$
f_{\partial B_{r}\left(x_{0}\right)} v \geq c(\kappa)\left(1-\frac{R^{N-2}}{\left(R+\frac{1-\kappa}{2} r\right)^{N-2}}\right) \geq c(N, \kappa, R) r,
$$

in contradiction with (5.7) if $c$ is small enough depending on $\kappa, N, R$.

Now we turn to the proof of the lemma for both cases. Since $x_{0}$ is fixed we simply write $B_{r}$ for $B_{r}\left(x_{0}\right)$. The idea is to take as a variation a domain, defined by a function coinciding with $v$ everywhere outside $B_{\sqrt{\kappa} r}$ and being zero inside $B_{\kappa r}$. More precisely, define $w$ in $B_{\sqrt{\kappa} r}$ as the solution of

$$
\left\{\begin{array}{l}
\Delta w=0 \text { in } B_{\sqrt{\kappa} r} \backslash B_{\kappa r} \\
w=0 \text { in } B_{\kappa r} \\
w=\bar{v} \text { on } \partial B_{\sqrt{\kappa} r}
\end{array},\right.
$$

where $\bar{v}=\sup _{B_{\sqrt{\kappa} r}} v$. Note that since $v$ is subharmonic, $\bar{v} \leq c(N, \kappa) f_{\partial B_{r}} v$. Moreover, one easily estimates

$$
\left|\frac{\partial w}{\partial \nu}\right| \leq C(n, \kappa) \frac{\bar{v}}{r} \quad \text { on } \partial B_{\kappa r} .
$$

Using the second inequality in Lemma 5.5 with $A=B_{\sqrt{\kappa} r}$ and $\max (u, 1-w)=1-\min (v, w)$ in the place of $w$, we get

$$
\int_{B_{\sqrt{\kappa} r}}|\nabla v|^{2} d x+\frac{\hat{\eta}}{2}\left|B_{\sqrt{\kappa} r} \cap\{v>0, w=0\}\right| \leq \int_{B_{\sqrt{\kappa} r}}|\nabla \min (v, w)|^{2} d x .
$$

Using Cauchy-Schwarz inequality, we obtain

$$
\begin{aligned}
& \int_{B_{\kappa r}}\left(|\nabla v|^{2}+\frac{\hat{\eta}}{2} 1_{\{v>0\}}\right) d x \leq \int_{B_{\sqrt{\kappa} r} \backslash B_{\kappa}}\left(|\nabla \min (v, w)|^{2}-|\nabla v|^{2}\right) d x \\
& \quad \leq 2 \int_{\left(B_{\sqrt{\kappa} r} \backslash B_{\kappa}\right) \cap\{v>w\}}\left(|\nabla w|^{2}-\nabla v \cdot \nabla w\right) d x=-2 \int_{B_{\sqrt{\kappa} r} \backslash B_{\kappa}} \nabla \max (v-w, 0) \nabla w d x \\
& \quad=2 \int_{\partial B_{\kappa r}} v \frac{\partial w}{\partial \nu} d \mathcal{H}^{N-1} \leq c(N, \kappa) \frac{\bar{v}}{r} \int_{\partial B_{\kappa r}} v d \mathcal{H}^{N-1} .
\end{aligned}
$$

where we have used (5.8). We will now bound $\int_{\partial B_{\kappa r}} v d \mathcal{H}^{N-1}$ from above by a constant times the left-hand side. Since $\frac{\bar{v}}{r}$ can be made as small as we wish, this will conclude the proof. In order to do that we use first the trace inequality, then Cauchy-Schwarz to get

$$
\begin{aligned}
\int_{\partial B_{\kappa r}} v d \mathcal{H}^{N-1} & \leq c(N, \kappa)\left(\frac{1}{r} \int_{B_{\kappa r}} v d x+\int_{B_{\kappa r}}|\nabla v| d x\right) \\
& \leq c(N, \kappa, R) \int_{B_{\kappa r}}\left(|\nabla v|^{2}+\frac{\hat{\eta}}{2} 1_{\{v>0\}}\right) d x .
\end{aligned}
$$


Lemma 5.10. There exists $M=M(N, R)$ such that if $u_{j}$ is a minimizer for (4.4) and $v_{j}=1-u_{j}$ satisfies

$$
f_{\partial B_{r}\left(x_{0}\right)} v_{j} d \mathcal{H}^{N-1} \geq M r
$$

then $v_{j}>0$ in $B_{r}\left(x_{0}\right)$.

Proof. Let us drop the subscript $j$ as usual. As a comparison domain here we consider $\Omega \backslash B_{r}\left(x_{0}\right)$, note that it is a subset of $B_{R}$.More precisely, we define $w$ as the solution of

$$
\left\{\begin{array}{l}
\Delta w=0 \text { in } B_{r}\left(x_{0}\right) \\
w=v \text { on } \mathbb{R}^{N} \backslash B_{r}\left(x_{0}\right) .
\end{array}\right.
$$

We use Lemma 5.5 and Remark 5.6 with $A=B_{r}, 1-w$ as $w$ to deduce

$$
\int_{B_{r}\left(x_{0}\right)}|\nabla(v-w)|^{2} d x \leq\left(\frac{1}{\hat{\eta}}+C \sigma\right)\left|\{v=0\} \cap B_{r}\left(x_{0}\right)\right| .
$$

We now estimate $\left|\{v=0\} \cap B_{r}\right|$ by the left-hand side. This can be done by arguing as in [AC81, Lemma 3.2]. Here we present a slightly different proof ${ }^{3}$. First we change coordinates so that $x_{0}=0$. Then by the representation formula

$$
w(x) \geq c(N) \frac{r-|x|}{r} f_{\partial B_{r}} v \geq c(N)(r-|x|) M .
$$

If we now apply Hardy inequality,

$$
\int_{B_{r}} \frac{g^{2}}{(r-|x|)^{2}} \leq C(N) \int_{B_{r}}|\nabla g|^{2} \quad g \in W_{0}^{1,2}\left(B_{r}\right),
$$

to the function $g=v-w$ and we take into account (5.10) and (5.9), we get

$$
\begin{aligned}
c(N) M^{2}\left|\{v=0\} \cap B_{r}\right| & \leq \int_{\{v=0\} \cap B_{r}} \frac{w^{2}}{(r-|x|)^{2}} \leq \int_{B_{r}} \frac{(w-v)^{2}}{(r-|x|)^{2}} \\
& \leq c(N) \int_{B_{r}}|\nabla(v-w)|^{2} \leq C(n, R)\left|\{v=0\} \cap B_{r}\right|,
\end{aligned}
$$

which is impossible if $M$ is large enough depending in $N, R$ unless $v>0$ almost everywhere in $B_{r}$.

As in Section 3 of [AC81] these two lemmas imply Lipschitz continuity of minimizers and density estimates on the boundary of minimizing domains. Note that we use here Lemma 5.8 as we need to apply the lemmas for the balls of all radii less or equal to some $\rho_{0}$, see (5.6).

Lemma 5.11. Let $v_{j}$ be as above, $\Omega_{j}=\left\{v_{j}=0\right\}$. Then $\Omega_{j}$ is open and there exist constants $C=C(N, R), \rho_{0}=\rho_{0}(N, R)>0$ such that

(i) for every $x \in B_{R}$

$$
\frac{1}{C} \operatorname{dist}\left(x, \Omega_{j}\right) \leq v_{j} \leq C \operatorname{dist}\left(x, \Omega_{j}\right) ;
$$

(ii) $v_{j}$ are equi-Lipschitz;

\footnotetext{
${ }^{3}$ We warmly thank Jonas Hirsch for suggesting this proof.
} 
(iii) for every $x \in \partial \Omega_{j}$ and $r \leq \rho_{0}$

$$
\frac{1}{C} \leq \frac{\left|\Omega_{j} \cap B_{r}(x)\right|}{\left|B_{r}(x)\right|} \leq\left(1-\frac{1}{C}\right) .
$$

Applying [AC81, Theorem 4.5] to $v_{j}=\left(1-u_{j}\right)$ we also have the following

Lemma 5.12. Let $u_{j}$ be as above, then there exists a Borel function $q_{u_{j}}$ such that

$$
\Delta u_{j}=q_{u_{j}} \mathcal{H}^{N-1}\left\llcorner\partial^{*} \Omega_{j} .\right.
$$

Moreover, $0<c \leq-q_{u_{j}} \leq C, c=c(n, R), C=C(n, R)$ and $\mathcal{H}^{N-1}\left(\partial \Omega_{j} \backslash \partial^{*} \Omega_{j}\right)=0$.

Since $\Omega_{j}$ converge to $B_{1}$ in $L^{1}$ by Lemma 4.4 , the density estimates also give us the following convergence of boundaries.

Lemma 5.13. Let $\Omega_{j}$ be minimizers of (4.4). Then:

(A) For the capacity with respect to the ball $B_{R}$

$$
\partial \Omega_{j} \underset{j \rightarrow \infty}{\longrightarrow} \partial B_{1}
$$

in the Kuratowski sense.

(B) For the capacity in $\mathbb{R}^{N}$ every limit point of $\Omega_{j}$ with respect to $L^{1}$ convergence is the unit ball centered at some $x_{\infty} \in B_{R}$. Moreover, the convergence holds also in the Kuratowski sense.

Corollary 5.14. In the setting of Lemma 5.13, for every $\delta>0$ there exists $j_{\delta}$ such that for $j \geq j_{\delta}$

(A) $B_{1-\delta} \subset \Omega_{j} \subset B_{1+\delta}$ in the case of the relative capacity;

(B) $B_{1-\delta}\left(x_{j}\right) \subset \Omega_{j} \subset B_{1+\delta}\left(x_{j}\right)$ for some $x_{j} \in B_{R}$ in the case of the capacity in $\mathbb{R}^{n}$.

5.2. Higher regularity of the free boundary. In order to address the higher regularity of $\partial \Omega_{j}$, we need to prove that $q_{u_{j}}$ is smooth. This will be done by using the Euler-Lagrange equations for our minimizing problem. We defined $\Omega_{j}$ in such a way that the following minimizing property holds

(A)

$$
\begin{aligned}
& \int_{B_{R}}\left|\nabla u_{j}\right|^{2} d x+f_{\hat{\eta}}\left(\left|\left\{u_{j}=1\right\}\right|\right)+\sqrt{\epsilon_{j}^{2}+\sigma^{2}\left(\alpha_{R}\left(\left\{u_{j}=1\right\}\right)-\epsilon_{j}\right)^{2}} \\
& \leq \int_{B_{R}}|\nabla u|^{2} d x+f_{\hat{\eta}}(|\{u=1\}|)+\sqrt{\epsilon_{j}^{2}+\sigma^{2}\left(\alpha_{R}(\{u=1\})-\epsilon_{j}\right)^{2}}
\end{aligned}
$$

for any $u \in W_{0}^{1,2}\left(B_{R}\right)$ such that $0 \leq u \leq 1$.

(B)

$$
\begin{aligned}
& \int_{\mathbb{R}^{N}}\left|\nabla u_{j}\right|^{2} d x+f_{\hat{\eta}}\left(\left|\left\{u_{j}=1\right\}\right|\right)+\sqrt{\epsilon_{j}^{2}+\sigma^{2}\left(\alpha\left(\left\{u_{j}=1\right\}\right)-\epsilon_{j}\right)^{2}} \\
& \leq \int_{\mathbb{R}^{N}}|\nabla u|^{2} d x+f_{\hat{\eta}}(|\{u=1\}|)+\sqrt{\epsilon_{j}^{2}+\sigma^{2}\left(\alpha(\{u=1\})-\epsilon_{j}\right)^{2}}
\end{aligned}
$$

for any $u \in W^{1,2}\left(\mathbb{R}^{N}\right)$ such that $0 \leq u \leq 1,\{u=1\} \subset B_{R}$.

To write Euler-Lagrange equations for $u_{j}$, we need to have (5.12) or (5.13))respectively for $u_{j} \circ \Phi$ where $\Phi$ is a diffeomorphism of $\mathbb{R}^{N}$ close to the identity. Note that to make sure that $\left\{u_{j} \circ \Phi=1\right\}$ is contained in $B_{R}$ one needs to know that $\operatorname{dist}\left(u_{j}, \partial B_{r}\right)>0$. This follows from Corollary 5.14, up translate $\Omega_{j}$ in the case of the absolute capacity (note that in this case the problem is invariant by translation). More precisely we will get the following optimality condition 
(A)

(B)

$$
q_{u_{j}}^{2}-\frac{\sigma^{2}\left(\alpha_{R}\left(\Omega_{j}\right)-\epsilon_{j}\right)}{\sqrt{\epsilon_{j}^{2}+\sigma^{2}\left(\alpha_{R}\left(\Omega_{j}\right)-\epsilon_{j}\right)^{2}}}|x|=\Lambda_{j}
$$

$$
q_{u_{j}}^{2}-\frac{\sigma^{2}\left(\alpha\left(\Omega_{j}\right)-\epsilon_{j}\right)}{\sqrt{\epsilon_{j}^{2}+\sigma^{2}\left(\alpha\left(\Omega_{j}\right)-\epsilon_{j}\right)^{2}}}\left(\left|x-x_{\Omega_{j}}\right|-\left(f_{\Omega_{j}} \frac{y-x_{\Omega_{j}}}{\left|y-x_{\Omega_{j}}\right|} d y\right) \cdot x\right)=\Lambda_{j}
$$

for some constant $\Lambda_{j}>0$. These equations are an immediate consequence of the following lemma whose proof is almost the same as [BDPV15, Lemma 4.15] (which in turn is based on [AAC86]). For this reason we only highlight the most relevant changes, referring the reader to [BDPV15, Lemma 4.15] for more details.

Lemma 5.15. There exists $j_{0}$ such that for any $j \geq j_{0}$ and any two points $x_{1}$ and $x_{2}$ in the reduced boundary of $\Omega_{j}$ the following equality holds:

(A)

$$
q_{u_{j}}^{2}\left(x_{1}\right)-\frac{\sigma^{2}\left(\alpha_{R}\left(\Omega_{j}\right)-\epsilon_{j}\right)}{\sqrt{\epsilon_{j}^{2}+\sigma^{2}\left(\alpha_{R}\left(\Omega_{j}\right)-\epsilon_{j}\right)^{2}}}\left|x_{1}\right|=q_{u_{j}}^{2}\left(x_{2}\right)-\frac{\sigma^{2}\left(\alpha_{R}\left(\Omega_{j}\right)-\epsilon_{j}\right)}{\sqrt{\epsilon_{j}^{2}+\sigma^{2}\left(\alpha_{R}\left(\Omega_{j}\right)-\epsilon_{j}\right)^{2}}}\left|x_{2}\right| ;
$$

$$
\begin{aligned}
q_{u_{j}}^{2}\left(x_{1}\right) & -\frac{\sigma^{2}\left(\alpha\left(\Omega_{j}\right)-\epsilon_{j}\right)}{\sqrt{\epsilon_{j}^{2}+\sigma^{2}\left(\alpha\left(\Omega_{j}\right)-\epsilon_{j}\right)^{2}}}\left(\left|x_{1}-x_{\Omega_{j}}\right|-\left(f_{\Omega_{j}} \frac{y-x_{\Omega_{j}}}{\left|y-x_{\Omega_{j}}\right|} d y\right) \cdot x_{1}\right) \\
& =q_{u_{j}}^{2}\left(x_{2}\right)-\frac{\sigma^{2}\left(\alpha\left(\Omega_{j}\right)-\epsilon_{j}\right)}{\sqrt{\epsilon_{j}^{2}+\sigma^{2}\left(\alpha\left(\Omega_{j}\right)-\epsilon_{j}\right)^{2}}}\left(\left|x_{2}-x_{\Omega_{j}}\right|-\left(f_{\Omega_{j}} \frac{y-x_{\Omega_{j}}}{\left|y-x_{\Omega_{j}}\right|} d y\right) \cdot x_{2}\right) .
\end{aligned}
$$

Proof. We argue by contradiction. Assume there exist $x_{1}, x_{2} \in \partial^{*}\left\{u_{j}=1\right\}$ such that

$$
q_{u_{j}}^{2}\left(x_{1}\right)-\frac{\sigma^{2}\left(\alpha_{R}\left(\Omega_{j}\right)-\epsilon_{j}\right)}{\sqrt{\epsilon_{j}^{2}+\sigma^{2}\left(\alpha_{R}\left(\Omega_{j}\right)-\epsilon_{j}\right)^{2}}}\left|x_{1}\right|<q_{u_{j}}^{2}\left(x_{2}\right)-\frac{\sigma^{2}\left(\alpha_{R}\left(\Omega_{j}\right)-\epsilon_{j}\right)}{\sqrt{\epsilon_{j}^{2}+\sigma^{2}\left(\alpha_{R}\left(\Omega_{j}\right)-\epsilon_{j}\right)^{2}}}\left|x_{2}\right|
$$

$$
\begin{aligned}
q_{u_{j}}^{2}\left(x_{1}\right) & -\frac{\sigma^{2}\left(\alpha\left(\Omega_{j}\right)-\epsilon_{j}\right)}{\sqrt{\epsilon_{j}^{2}+\sigma^{2}\left(\alpha\left(\Omega_{j}\right)-\epsilon_{j}\right)^{2}}}\left(\left|x_{1}-x_{\Omega_{j}}\right|-\left(f_{\Omega_{j}} \frac{y-x_{\Omega_{j}}}{\left|y-x_{\Omega_{j}}\right|} d y\right) \cdot x_{1}\right) \\
& <q_{u_{j}}^{2}\left(x_{2}\right)-\frac{\sigma^{2}\left(\alpha\left(\Omega_{j}\right)-\epsilon_{j}\right)}{\sqrt{\epsilon_{j}^{2}+\sigma^{2}\left(\alpha\left(\Omega_{j}\right)-\epsilon_{j}\right)^{2}}}\left(\left|x_{2}-x_{\Omega_{j}}\right|-\left(f_{\Omega_{j}} \frac{y-x_{\Omega_{j}}}{\left|y-x_{\Omega_{j}}\right|} d y\right) \cdot x_{2}\right) .
\end{aligned}
$$

Using this inequalities, we are going to construct a variation contradicting (5.12). We take a smooth radial symmetric function $\phi(x)=\phi(|x|)$ supported in $B_{1}$ and define the following diffeomorphism for small $\tau$ and $\rho$ :

$$
\Phi_{\tau}^{\rho}(x)= \begin{cases}x+\tau \rho \phi\left(\left|\frac{x-x_{1}}{\rho}\right|\right) \nu\left(x_{1}\right), & x \in B_{\rho}\left(x_{1}\right), \\ x-\tau \rho \phi\left(\left|\frac{\mid-x_{2}}{\rho}\right|\right) \nu\left(x_{2}\right), & x \in B_{\rho}\left(x_{2}\right), \\ x, & \text { otherwise. }\end{cases}
$$


We define the function

$$
u_{\tau}^{\rho}:=u \circ\left(\Phi_{\tau}^{\rho}\right)^{-1}
$$

and we define a competitor domain $\Omega_{\tau}^{\rho}$ as the domain with $u_{\tau}^{\rho}$ for capacitary potential, i.e.

$$
\Omega_{\tau}^{\rho}:=\left\{u_{\tau}^{\rho}=1\right\} .
$$

Now we are going to show that for $\tau$ and $\rho$ small enough $\mathscr{C}_{\hat{\eta}}^{*}\left(\Omega_{\tau}^{\rho}\right)<\mathscr{C}_{\hat{\eta}}^{*}(\Omega)$. To do that, we first compute the variation of all the terms involved in $\mathscr{C}_{\hat{\eta}}^{*}$.

Volume. By arguing as in [BDPV15, Lemma 4.15] one gets

$$
\begin{aligned}
\left|\Omega_{\tau}^{\rho}\right|-|\Omega| & =\tau \rho^{N}\left(\int_{\left\{y \cdot \nu\left(x_{1}\right)=0\right\} \cap B_{1}} \phi(|y|)-\int_{\left\{y \cdot \nu\left(x_{2}\right)=0\right\} \cap B_{1}} \phi(|y|)\right)+o(\tau) \rho^{N}+o_{\tau}\left(\rho^{N}\right) \\
& =o(\tau) \rho^{N}+o_{\tau}\left(\rho^{N}\right),
\end{aligned}
$$

where $o_{\tau}\left(\rho^{N}\right) \rho^{-N}$ goes to zero as $\rho \rightarrow 0$ and $o(\tau)$ is independent on $\rho$.

Barycenter. (for the case of the capacity in $\mathbb{R}^{N}$ ). Assume that that $x_{\Omega}=0$, as in [BDPV15, Lemma 4.15] one gets,

$$
x_{\Omega_{\tau}^{\rho}}=-\rho^{N} \tau \frac{x_{1}-x_{2}}{|\Omega|}\left(\int_{\left\{y_{1}=0\right\} \cap B_{1}} \phi(|y|)\right)+\rho^{N} o(\tau)+o_{\tau}\left(\rho^{N}\right) .
$$

Asymmetry. Again by the very same computations as in [BDPV15, Lemma 4.15] one gets

$$
\alpha_{R}\left(\Omega_{\tau}^{\rho}\right)-\alpha_{R}(\Omega)=-\rho^{N} \tau\left(\int_{\left\{y_{1}=0\right\} \cap B_{1}} \phi(|y|)\right)\left(\left|x_{1}\right|-\left|x_{2}\right|\right)+o(\tau) \rho^{N}+o_{\tau}\left(\rho^{N}\right) .
$$

In the case of asymmetry $\alpha(\Omega)$ we get an additional term:

$$
\alpha\left(\Omega_{\tau}^{\rho}\right)-\alpha(\Omega)=-\rho^{N} \tau\left(\int_{\left\{y_{1}=0\right\} \cap B_{1}} \phi(|y|)\right)\left(\left|x_{1}\right|-\left|x_{2}\right|+\left(f_{\Omega} \frac{y}{|y|} d y\right) \cdot\left(x_{1}-x_{2}\right)\right)+o(\tau) \rho^{N}+o_{\tau}\left(\rho^{N}\right) .
$$

Dirichlet energy. Again one can argue as in [BDPV15, Lemma 4.15] to get

$$
\operatorname{cap}_{*}\left(\Omega_{\tau}^{\rho}\right)-\operatorname{cap}_{*}(\Omega) \leq \tau \rho^{N}\left(\left|q\left(x_{1}\right)\right|^{2}-\left|q\left(x_{2}\right)\right|^{2}\right) \int_{B_{1} \cap\left\{y_{1}=0\right\}} \phi(|y|) d y+o(\tau) \rho^{N}+o_{\tau}\left(\rho^{N}\right) .
$$

Combining the above estimates one gets

(A)

$$
\begin{aligned}
& \left(\int_{B_{1} \cap\left\{y_{1}=0\right\}} \phi(|y|) d y\right)^{-1} \frac{\mathscr{C}_{\hat{\eta}, j}^{R}\left(\Omega_{\tau}^{\rho}\right)-\mathscr{C}_{\hat{\eta}, j}^{R}(\Omega)}{\rho^{N}} \\
& =\tau\left(\left|q\left(x_{1}\right)\right|^{2}-\left|q\left(x_{2}\right)\right|^{2}-\frac{\sigma^{2}\left(\alpha_{R}(\Omega)-\epsilon_{j}\right)}{\sqrt{\epsilon_{j}^{2}+\sigma^{2}\left(\alpha_{R}(\Omega)-\epsilon_{j}\right)^{2}}}\left(\left|x_{1}\right|-\left|x_{2}\right|\right)\right)+o(\tau)+o_{\tau}(1) ;
\end{aligned}
$$


(B)

$$
\begin{aligned}
& \left(\int_{B_{1} \cap\left\{y_{1}=0\right\}} \phi(|y|) d y\right)^{-1} \frac{\mathscr{C}_{\hat{\eta}, j}\left(\Omega_{\tau}^{\rho}\right)-\mathscr{C}_{\hat{\eta}, j}(\Omega)}{\rho^{N}} \\
& =\tau\left(\left|q\left(x_{1}\right)\right|^{2}-\left|q\left(x_{2}\right)\right|^{2}-\frac{\sigma^{2}\left(\alpha(\Omega)-\epsilon_{j}\right)}{\sqrt{\epsilon_{j}^{2}+\sigma^{2}\left(\alpha(\Omega)-\epsilon_{j}\right)^{2}}}\left(\left|x_{1}\right|-\left|x_{2}\right|+\left(f_{\Omega} \frac{y}{|y|} d y\right) \cdot\left(x_{1}-x_{2}\right)\right)\right) \\
& \quad+o(\tau)+o_{\tau}(1) .
\end{aligned}
$$

According to (5.14) and (5.15) the quantity in parentheses is strictly negative. Thus, we get a contradiction with the minimality of $\Omega$ for $\rho$ and $\tau$ small enough.

Lemma 5.16 (Smoothness of $q_{u}$ ). There exist constants $\delta=\delta(N, R)>0, j_{0}=j_{0}(N, R)$, $\sigma_{0}=\sigma_{0}(N, R)>0$ such that for every $j \geq j_{0}, \sigma \leq \sigma_{0}$ the functions $q_{u_{j}}$ belong to $C^{\infty}\left(\mathcal{N}_{\delta}\left(\partial \Omega_{j}\right)\right)$.

Moreover, for every $k$ there exists a constant $C=C(k, N, R)$ such that

$$
\left\|q_{u_{j}}\right\|_{C^{k}\left(\mathcal{N}_{\delta}\left(\partial \Omega_{j}\right)\right)} \leq C
$$

for every $j \geq j_{0}$.

Proof. We would like to write an explicit formula for $q_{u_{j}}$ using Euler-Lagrange equations, namely

(A)

$$
q_{u_{j}}=-\left(\frac{\sigma^{2}\left(\alpha_{R}\left(\Omega_{j}\right)-\epsilon_{j}\right)}{\sqrt{\epsilon_{j}^{2}+\sigma^{2}\left(\alpha_{R}\left(\Omega_{j}\right)-\epsilon_{j}\right)^{2}}}|x|+\Lambda_{j}\right)^{\frac{1}{2}}
$$

(B)

$$
q_{u_{j}}=-\left(\frac{\sigma^{2}\left(\alpha\left(\Omega_{j}\right)-\epsilon_{j}\right)}{\sqrt{\epsilon_{j}^{2}+\sigma^{2}\left(\alpha\left(\Omega_{j}\right)-\epsilon_{j}\right)^{2}}}\left(\left|x-x_{\Omega_{j}}\right|-\left(f_{\Omega_{j}} \frac{y-x_{\Omega_{j}}}{\left|y-x_{\Omega_{j}}\right|} d y\right) \cdot x\right)+\Lambda_{j}\right)^{\frac{1}{2}} .
$$

To do that, we need to show that the quantity in the parenthesis is bounded away from zero. Indeed, $q_{u_{j}}$ is bounded from above and below independently of $j$ and

$$
\left|\frac{\sigma^{2}\left(\alpha_{R}\left(\Omega_{j}\right)-\epsilon_{j}\right)}{\sqrt{\epsilon_{j}^{2}+\sigma^{2}\left(\alpha_{R}\left(\Omega_{j}\right)-\epsilon_{j}\right)^{2}}}\right| x|| \leq C(N, R) \sigma
$$

$$
\left|\frac{\sigma^{2}\left(\alpha\left(\Omega_{j}\right)-\epsilon_{j}\right)}{\sqrt{\epsilon_{j}^{2}+\sigma^{2}\left(\alpha\left(\Omega_{j}\right)-\epsilon_{j}\right)^{2}}}\left(\left|x-x_{\Omega_{j}}\right|-\left(f_{\Omega_{j}} \frac{y-x_{\Omega_{j}}}{\left|y-x_{\Omega_{j}}\right|} d y\right) \cdot x\right)\right| \leq C(N, R) \sigma .
$$

Then it follows from the Euler-Lagrange equations that also $\Lambda_{j}$ is bounded from above and below independently of $j$. Thus, for $\sigma$ small enough we can write the above-mentioned explicit formula for $q_{u_{j}}$ and get the conclusion of the lemma.

Now we are ready to apply the results of [AC81]. Indeed thanks to Lemma 5.15, $v_{j}=$ $\left(1-u_{j}\right)$ is a weak solution of the free boundary problem First, we need to recall the 
definition of flatness for the free boundary, see [AC81, Definition 7.1] (here it is applied to $u=(1-v))$.

Definition 5.17. Let $\mu_{-}, \mu_{+} \in(0,1]$. A weak solution $u$ of $(5.11)$ is said to be of class $F\left(\mu_{-}, \mu_{+}, \infty\right)$ in $B_{\rho}\left(x_{0}\right)$ in a direction $\nu \in S^{N-1}$ if $x_{0} \in \partial\{u=1\}$ and

$$
\begin{cases}u(x)=1 & \text { for }\left(x-x_{0}\right) \cdot \nu \leq-\mu_{-} \rho, \\ 1-u(x) \geq q_{u}\left(x_{0}\right)\left(\left(x-x_{0}\right) \cdot \nu-\mu_{+} \rho\right) & \text { for }\left(x-x_{0}\right) \cdot \nu \geq \mu_{+} \rho,\end{cases}
$$

We are going to use that flat free boundaries are smooth (again we apply [AC81, Theorem 8.1] to $v=(1-u))$

Theorem 5.18 (Theorem 8.1 in [AC81]). Let u be a weak solution of (5.11)) and assume that $q_{u}$ is Lipschitz continuous. There are constants $\gamma, \mu_{0}, \kappa, C$ such that if $u$ is of class $F(\mu, 1, \infty)$ in $B_{4 \rho}\left(x_{0}\right)$ in some direction $\nu \in S^{N-1}$ with $\mu \leq \mu_{0}$ and $\rho \leq \kappa \mu^{2}$, then there exists a $C^{1, \gamma}$ function $f: \mathbb{R}^{N-1} \rightarrow \mathbb{R}$ with $\|f\|_{C^{1, \gamma}} \leq C \mu$ such that

$$
\partial\{u=1\} \cap B_{\rho}\left(x_{0}\right)=\left(x_{0}+\operatorname{graph}_{\nu} f\right) \cap B_{\rho}\left(x_{0}\right),
$$

where $\left.\operatorname{graph}_{\nu} f=\left\{x \in \mathbb{R}^{N}: x \cdot \nu=f(x-x \cdot \nu) \nu\right)\right\}$. Moreover if $q_{u} \in C^{k, \gamma}$ in some neighborhood of $\left\{u_{j}=1\right\}$, then $f \in C^{k+1, \gamma}$ and $\|f\|_{C^{k+1, \gamma}} \leq C\left(N, R,\left\|q_{u}\right\|_{C^{k, \gamma}}\right)$.

We are now ready to prove Theorem 3.2, cp. [BDPV15, Proposition 4.4].

Proof of Theorem 3.2. We define $\Omega_{j}$ as minimizers of (4.4). The desired sequence of Selection Principle will be properly rescaled $\left\{\Omega_{j}\right\}$. We need to show that $\left\{\Omega_{j}\right\}$ converges smoothly to the ball $B_{1}$. Indeed one then define

$$
U_{j}=\lambda_{j}\left(\Omega_{j}-x_{*}\right),
$$

where $x_{*}=0$ in the case of the relative capacity and $x_{*}=x_{\Omega_{j}}$ in the case of the absolute capacity. Theorem 4.4 then implies all the desired properties of $U_{j}$, compare with [BDPV15, Proof of Proposition 4.4].

Let $\mu_{0}, \kappa$ be as in Theorem 5.18 and $\mu<\mu_{0}$ to be fixed later. Let $\bar{x}$ be some point on the boundary of $B_{1}$. As $\partial B_{1}$ is smooth, it lies inside a narrow strip in the neighborhood of $\bar{x}$. More precisely, there exists $\rho_{0}=\rho_{0}(\mu) \leq \kappa \mu^{2}$ such that for every $\rho<\rho_{0}$ and every $\bar{x} \in \partial B_{1}$

$$
\partial B_{1} \cap B_{5 \rho}(\bar{x}) \subset\left\{x:\left|(x-\bar{x}) \cdot \nu_{\bar{x}}\right| \leq \mu \rho\right\} .
$$

We know that $\partial \Omega_{j}$ are converging to $\partial B_{1}$ in the sense of Kuratowski. Thus, there exists a point $x_{0} \in \partial \Omega_{j} \cap B_{\mu \rho_{0}}(\bar{x})$ such that

$$
\partial \Omega_{j} \cap B_{4 \rho_{0}}\left(x_{0}\right) \subset\left\{x:\left|\left(x-x_{0}\right) \cdot \nu_{\bar{x}}\right| \leq 4 \mu \rho_{0}\right\} .
$$

So, $u_{j}$ is of class $F(\mu, 1, \infty)$ in $B_{4 \rho_{0}}\left(x_{0}\right)$ with respect to the direction $\nu_{\bar{x}}$ and by Theorem 5.18, $\partial \Omega_{j} \cap B_{\rho_{0}}\left(x_{0}\right)$ is the graph of a smooth function with respect to $\nu_{\bar{x}}$. More precisely, for $\mu$ small enough there exists a family of smooth functions $g_{j}^{\bar{x}}$ with uniformly bounded $C^{k}$ norms such that

$$
\partial \Omega_{j} \cap B_{\rho_{0}}(\bar{x})=\left\{x+g_{j}^{\bar{x}}(x) x: x \in \partial B_{1}\right\} \cap B_{\rho_{0}}(\bar{x}) .
$$

By a covering argument this gives a family of smooth functions $g_{j}$ with uniformly bounded $C^{k}$ norms such that

$$
\partial \Omega_{j}=\left\{x+g_{j}(x) x: x \in \partial B_{1}\right\} .
$$

By Ascoli-Arzelà and convergence to $\partial B_{1}$ in the sense of Kuratowski, we get that $g_{j} \rightarrow 0$ in $C^{k-1}\left(\partial B_{1}\right)$, hence the smooth convergence of $\partial \Omega_{j}$. 


\section{ReduCtion to BOUnded SETS}

To complete the proof of Theorem 1.4 one needs to show that in the case of the full capacity one can just consider sets with uniformly bounded diameter. To this end let us introduce the following

Definition 6.1. Let $\Omega$ be an open set in $\mathbb{R}^{n}$ with $|\Omega|=\left|B_{1}\right|$. Then we define the deficit of $\Omega$ as the difference between its capacity and the capacity of the unit ball:

$$
D(\Omega)=\operatorname{cap}(\Omega)-\operatorname{cap}\left(B_{1}\right) .
$$

Here is the key lemma for reducing Theorem 1.4 to Theorem 3.1.

Lemma 6.2. There exist constants $C=C(N), \delta=\delta(N)>0$ and $d=d(N)$ such that for any $\Omega \subset \mathbb{R}^{n}$ open with $|\Omega|=\left|B_{1}\right|$ and $D(\Omega) \leq \delta$, we can find a new set $\tilde{\Omega}$ enjoying the following properties

(1) $\operatorname{diam}(\tilde{\Omega}) \leq d$,

(2) $|\tilde{\Omega}|=\left|B_{1}\right|$,

(3) $D(\tilde{\Omega}) \leq C D(\Omega)$,

(4) $\mathcal{A}(\tilde{\Omega}) \geq \mathcal{A}(\Omega)-C D(\Omega)$.

We are going to define $\tilde{\Omega}$ as a suitable dilation of $\Omega \cap B_{S}$ for some large $S$. Hence, we first show the following estimates on the capacity of $\Omega \cap B_{S}$.

Lemma 6.3. Let $S^{\prime}>S$. Then there exists a constant $c=c\left(S^{\prime}\right)$ such that for any open set $\Omega \subset \mathbb{R}^{N}$ with $|\Omega|=\left|B_{1}\right|$ the following inequalities hold:

$$
\operatorname{cap}\left(B_{1}\right)\left(1-\frac{\left|\Omega \backslash B_{S}\right|}{\left|B_{1}\right|}\right)^{\frac{N-2}{N}} \leq \operatorname{cap}\left(\Omega \cap B_{S}\right) \leq \operatorname{cap}(\Omega)-c\left(1-\frac{S}{S^{\prime}}\right)^{\frac{N-2}{N}}\left|\Omega \backslash B_{S^{\prime}}\right|^{\frac{N-2}{N}} .
$$

Proof. The first inequality is a direct consequence of the classical isocapacitary inequality. To prove the second one we are going to use the estimates for the capacitary potential of $B_{S}$ for which the exact formula can be written. Denote by $u_{\Omega}$ and $u_{S}$ the capacitary potentials of $\Omega$ and $\Omega \cap B_{S}$ respectively. We first compute

$$
\begin{aligned}
& \operatorname{cap}\left(\Omega \cap B_{S}\right)=\operatorname{cap}(\Omega)+\int_{\mathbb{R}^{n}}\left|\nabla u_{S}\right|^{2}-\left|\nabla u_{\Omega}\right|^{2} \\
& =\operatorname{cap}(\Omega)-\int_{\left(\Omega \cap B_{S}\right)^{c}}\left|\nabla\left(u_{\Omega}-u_{S}\right)\right|^{2}+2 \int_{\left(\Omega \cap B_{S}\right)^{c}} \nabla u_{S} \cdot \nabla\left(u_{S}-u_{\Omega}\right) \\
& =\operatorname{cap}(\Omega)-\int_{\left(\Omega \cap B_{S}\right)^{c}}\left|\nabla\left(u_{\Omega}-u_{S}\right)\right|^{2}-2 \int_{\left(\Omega \cap B_{S}\right)^{c}}\left(\Delta u_{S}\right)\left(u_{S}-u_{\Omega}\right) \\
& \quad+2 \int_{\partial\left(\Omega \cap B_{S}\right)}\left(u_{S}-u_{\Omega}\right) \nabla u_{S} \cdot \nu d \mathcal{H}^{N-1} \\
& =\operatorname{cap}(\Omega)-\int_{\left(\Omega \cap B_{S}\right)^{c}}\left|\nabla\left(u_{\Omega}-u_{S}\right)\right|^{2}
\end{aligned}
$$

since $u_{S}=u_{\Omega}=1$ on $\partial\left(\Omega \cap B_{S}\right)$. We would like to show that $\int_{\left(\Omega \cap B_{S}\right)^{c}}\left|\nabla\left(u_{\Omega}-u_{S}\right)\right|^{2}$ cannot be too small. To this end let us set $v_{\Omega}=1-u_{\Omega}$ and similarly for $v_{S}$. By Sobolev's embedding we get

$$
\begin{aligned}
& \int_{\left(\Omega \cap B_{S}\right)^{c}}\left|\nabla\left(u_{\Omega}-u_{S}\right)\right|^{2}=\int_{\left(\Omega \cap B_{S}\right)^{c}}\left|\nabla\left(v_{\Omega}-v_{S}\right)\right|^{2} \\
& \geq c(N)\left(\int_{\left(\Omega \cap B_{S}\right)^{c}}\left|v_{\Omega}-v_{S}\right|^{2^{*}}\right)^{\frac{2}{2^{*}}} \geq c\left(\int_{\Omega \backslash B_{S}}\left|v_{S}\right|^{2^{*}}\right)^{\frac{2}{2^{*}}},
\end{aligned}
$$


where $2^{*}$ is the Sobolev exponent and in the last inequality we used that $v_{\Omega} \equiv 0$ on $\Omega$. Let us also set

$$
z_{S}=\left(1-\frac{S^{N-2}}{|x|^{N-2}}\right)_{+}
$$

By the maximum principle, $v_{S} \geq z_{S}$, hence

$$
\begin{aligned}
\int_{\Omega \backslash B_{S}}\left|v_{S}\right|^{2^{*}} & \geq \int_{\Omega \backslash B_{S}}\left|z_{S}\right|^{2^{*}} \\
& \geq \int_{\Omega \backslash B_{S^{\prime}}}\left|z_{S}\right|^{2^{*}} \geq\left(1-\left(\frac{S}{S^{\prime}}\right)^{N-2}\right)^{\frac{2 N}{N-2}}\left|\Omega \backslash B_{S^{\prime}}\right| .
\end{aligned}
$$

Hence

$$
\begin{aligned}
\operatorname{cap}\left(\Omega \cap B_{S}\right) & \leq \operatorname{cap}(\Omega)-c(N)\left(1-\left(\frac{S}{S^{\prime}}\right)^{N-2}\right)^{\frac{N-2}{N}}\left|\Omega \backslash B_{S^{\prime}}\right|^{\frac{N-2}{N}} \\
& \leq \operatorname{cap}(\Omega)-c\left(1-\frac{S}{S^{\prime}}\right)^{\frac{N-2}{N}}\left|\Omega \backslash B_{S^{\prime}}\right|^{\frac{N-2}{N}}
\end{aligned}
$$

concluding the proof.

We can now prove Lemma 6.2.

Proof of Lemma 6.2. . Let us assume without loss of generality that the ball achieving the asymmetry of $\Omega$ is $B_{1}$. As was already mentioned, we are going to show that there exists an $\tilde{\Omega}$ of the form $\lambda\left(\Omega \cap B_{S}\right)$ for suitable $S$ and $\lambda$ satisfying all the desired properties. Let us set

$$
b_{k}:=\frac{\left|\Omega \backslash B_{2-2^{k}}\right|}{\left|B_{1}\right|} \leq 1 .
$$

Note that by Theorem 1.3 we can assume that $b_{1} \leq 2 \mathcal{A}(\Omega)$ is as small as we wish (independently on $\Omega$ up to choose $\delta$ sufficiently small. Lemma 6.3 gives

$$
\operatorname{cap}(\Omega)-c\left(\frac{2^{-(k+1)}}{2-2^{-(k+1)}}\right)^{\frac{N-2}{N}} b_{k+1}^{\frac{N-2}{N}} \geq \operatorname{cap}\left(B_{1}\right)\left(1-b_{k}\right)^{\frac{N-2}{N}} \geq \operatorname{cap}\left(B_{1}\right)-\operatorname{cap}\left(B_{1}\right) b_{k},
$$

which implies

$$
c b_{k+1} \leq 2^{k}\left(D(\Omega)+C b_{k}\right)^{\frac{N}{N-2}} .
$$

We now claim that there exists $\bar{k}$ such that

$$
b_{\bar{k}} \leq D(\Omega) .
$$

Indeed, otherwise by (6.1) we wpuld get

$$
b_{k+1} \leq C 2^{k}\left(D(\Omega)+C b_{k}\right)^{\frac{N}{N-2}} \leq 2^{k} C^{\prime} b_{k}^{\frac{N}{N-2}} \leq M^{k} b_{k}^{\frac{N}{N-2}}
$$

for all $k \in \mathbb{N}$, where $M=M(N)$. Iterating the last inequality, we obtain

$$
b_{k+1} \leq\left(M b_{1}\right)^{\left(\frac{N}{N-2}\right)^{k}} \underset{k \rightarrow \infty}{\longrightarrow} 0
$$

if $b_{1}$ is small enough, which by Theorem 1.3 we can assume up to choose $\delta=\delta(N) \ll 1$.

We define $\tilde{\Omega}$ as a properly rescaled intersection of $\Omega$ with a ball. Let $\bar{k}$ be such that $b_{\bar{k}} \leq D(\Omega)$

$$
\tilde{\Omega}:=\left(\frac{\left|B_{1}\right|}{\left|\Omega \cap B_{R}\right|}\right)^{\frac{1}{N}}\left(\Omega \cap B_{R}\right)=\left(1-b_{\bar{k}}\right)^{-\frac{1}{N}}\left(\Omega \cap B_{S}\right),
$$


where $S:=2-2^{-\bar{k}} \leq 2$. Note that $|\tilde{\Omega}|=\left|B_{1}\right|$. We now check all the remaining properties:

- Bound on the diameter:

$$
\operatorname{diam}(\tilde{\Omega}) \leq 2 \cdot 2(1-D(\Omega))^{-\frac{1}{N}} \leq 4(1-\delta)^{-\frac{1}{N}} \leq 4 .
$$

up to choose $\delta=\delta(N) \ll 1$.

- Bound on the deficit:

$$
\begin{aligned}
D(\tilde{\Omega}) & =\operatorname{cap}(\tilde{\Omega})-\operatorname{cap}\left(B_{1}\right)=\operatorname{cap}\left(\Omega \cap B_{S}\right)\left(1-b_{\bar{K}}\right)^{-\frac{N-2}{N}}-\operatorname{cap}\left(B_{1}\right) \\
& \leq \operatorname{cap}(\Omega)\left(1-b_{\bar{k}}\right)^{-\frac{N-2}{N}}-\operatorname{cap}\left(B_{1}\right) \\
& \leq \operatorname{cap}(\Omega)-\operatorname{cap}\left(B_{1}\right)+\frac{2(N-2) \operatorname{cap}(\Omega)}{N} b_{\bar{k}} \leq C(N) D(\Omega) .
\end{aligned}
$$

since $b_{\bar{k}} \leq D(\Omega) \ll 1$ and, in particular, $\operatorname{cap}(\Omega) \leq 2 \operatorname{cap}\left(B_{1}\right)$.

- Bound on the asymmetry: Let $r:=\left(1-b_{\bar{k}}\right)^{-1} \in(1,2)$, that is $r$ is such that $\tilde{\Omega}=r^{N}\left(\Omega \cap B_{S}\right)$ with $S=2-2^{-\bar{k}} \leq 2$. Let $x_{0}$ be such that $B_{1}\left(x_{0}\right)$ is a minimizing ball for $\mathcal{A}(\tilde{\Omega})$. Then, recalling that $b_{\bar{k}}=\left|B_{1}\right|^{-1}\left|\Omega \backslash B_{S}\right| \leq C(N) D(\Omega)$,

$$
\begin{aligned}
\left|B_{1}\right| \mathcal{A}(\Omega) \leq & \left|\Omega \Delta B_{1}\left(\frac{x_{0}}{r}\right)\right| \leq\left|\Omega \backslash B_{S}\right|+\left|\left(\Omega \cap B_{S}\right) \Delta B_{1}\left(\frac{x_{0}}{r}\right)\right| \\
\leq & C D(\Omega)+\left|\left(\Omega \cap B_{S}\right) \Delta B_{\frac{1}{r}}\left(\frac{x_{0}}{r}\right)\right| \\
& +\left|B_{\frac{1}{r}}\left(\frac{x_{0}}{r}\right) \Delta B_{1}\left(\frac{x_{0}}{r}\right)\right| \\
\leq & C D(\Omega)+\frac{\left|B_{1}\right|}{r^{N}} \mathcal{A}(\tilde{\Omega})+\left|B_{1}\right|\left(1-\frac{1}{r^{N}}\right) \\
\leq & C D(\Omega)+\left|B_{1}\right| \mathcal{A}(\tilde{\Omega})+C(N) b_{\bar{k}} \\
\leq & C D(\Omega)+\left|B_{1}\right| \mathcal{A}(\tilde{\Omega}) .
\end{aligned}
$$

\section{Proof of Theorem 1.4}

In order to reduce it to Theorem 3.1 we need to start with a set which is already close to a ball. In the case of the absolute capacity, thanks to Theorem 1.3, this can be achieved by assuming the deficit sufficiently small (the quantitative inequality being trivial in the other regime). The next lemma contains the same "qualitative" result in the case of the relative capacity.

Lemma 7.1. For all $\varepsilon>0$ there exists $\delta=\delta(\varepsilon, R)>0$ such that if $\Omega \subset B_{R}$ is an open set with $|\Omega|=1$ and

then

$$
\operatorname{cap}_{R}(\Omega) \leq \operatorname{cap}_{R}\left(B_{1}\right)+\delta
$$

$$
\alpha_{R}(\Omega) \leq \varepsilon .
$$

Proof. We argue by contradiction. Suppose there exists an $\varepsilon_{0}>0$ and a sequence of open sets $\Omega_{j} \subset B_{R}$ with $\left|\Omega_{j}\right|=\left|B_{1}\right|$ such that $\alpha_{R}\left(\Omega_{j}\right) \geq \varepsilon_{0}$ but

$$
\operatorname{cap}_{R}\left(B_{1}\right) \leq \operatorname{cap}_{R}\left(\Omega_{j}\right) \leq \operatorname{cap}_{R}\left(B_{1}\right)+1 / j .
$$

We denote by $u_{j} \in W_{0}^{1,2}\left(B_{R}\right)$ the capacitary potential of $\Omega_{j}$. The above inequality grants that

$$
\int_{B_{R}}\left|\nabla u_{j}\right|^{2} d x \rightarrow \operatorname{cap}_{R}\left(B_{1}\right) .
$$


Thus, up to a not-relabelled subsequence, there exists a function $u$ in $W_{0}^{1,2}\left(B_{R}\right)$ such that $u_{j} \rightarrow u$ in $W_{0}^{1,2}\left(B_{R}\right), u_{j} \rightarrow u$ in $L^{2}\left(B_{R}\right)$ and almost everywhere in $B_{R}$. We define $\Omega$ as $\{u=1\}$. From the lower semi-continuity of Dirichlet integral we have that

$$
\operatorname{cap}_{R}(\Omega) \leq \int_{B_{R}}|\nabla u|^{2} d x \leq \liminf \int_{B_{R}}\left|\nabla u_{j}\right|^{2} d x=\operatorname{cap}_{R}\left(B_{1}\right) .
$$

On the other hand, we have $1_{\Omega} \geq \limsup 1_{\Omega_{j}}$, meaning that $\left|\Omega_{j} \backslash \Omega\right| \rightarrow 0$ and $|\Omega| \geq\left|\Omega_{j}\right|=$ $\left|B_{1}\right|$. The isocapacitary inequality then implies that $\Omega=B_{1}$. In particular, $\left|\Omega_{j}\right|=|\Omega|$ for all $j$ and

$$
\left|\Omega \backslash \Omega_{j}\right|=\left|\Omega_{j} \backslash \Omega\right| \rightarrow 0,
$$

and thus $1_{\Omega_{j}} \rightarrow 1_{\Omega}=1_{B_{1}}$ in $L^{1}\left(B_{R}\right)$. Hence by Lemma 2.3, (ii), $\alpha_{R}\left(\Omega_{j}\right) \rightarrow 0$, a contradiction.

We have now all the ingredients to prove Theorem 1.4.

Proof of Theorem 1.4. We will consider separately the cases of the absolute and relative capacity.

Absolute capacity. First note that if $D(\Omega) \geq \delta_{0}$ then, since $\mathcal{A}(\Omega) \geq 2$,

$$
D(\Omega) \geq 4 \frac{\delta_{0}}{4} \geq \frac{\delta_{0}}{4} \mathcal{A}(\Omega)^{2} .
$$

Hence we can assume that $D(\Omega)$ is as small as we wish as long as the smallness depends only on $N$. We now $\delta_{0}$ smaller than the constant $\delta$ in Lemma 6.2 and, assuming that $D(\Omega) \leq \delta_{0}$, we use Lemma 6.2 to find a set $\tilde{\Omega}$ with $\operatorname{diam}(\tilde{\Omega}) \leq d=d(N)$ and satisfying all the properties there. In particular, up to a translation we can assume that $\tilde{\Omega} \subset B_{d}$. Up to choosing $\delta_{0}$ smaller we can apply Theorem 1.3 and Lemma 2.3 (ii) to ensure that $\alpha(\tilde{\Omega}) \leq \epsilon_{0}$ where $\epsilon_{0}=\epsilon_{0}(N, d)=\epsilon_{0}(N)$ is the constant appearing in the statement of Theorem 3.1. This, together with Lemma 2.3, (i), grants that

$$
D(\tilde{\Omega}) \geq c(N) \alpha(\tilde{\Omega}) \geq c(N) \mathcal{A}(\tilde{\Omega})^{2} .
$$

Hence, by Lemma 6.2 and assuming that $\mathcal{A}(\Omega) \geq C D(\Omega)$ (since otherwise there is nothing to prove),

$$
D(\Omega) \geq c D(\tilde{\Omega}) \geq c A(\tilde{\Omega})^{2} \geq c \mathcal{A}(\tilde{\Omega})^{2} \geq c \mathcal{A}(\Omega)^{2}-C D(\Omega)^{2}
$$

from which the conclusion easily follows since $D(\Omega) \leq \delta_{0} \ll 1$.

Relative capacity. Since $\alpha_{R}(\Omega) \leq C(R, N)$ by arguing as in the previous case, we can assume that $\operatorname{cap}_{R}(\Omega)-\operatorname{cap}_{R}\left(B_{1}\right) \leq \delta_{1}(N, R) \ll 1$. By Lemma 7.1 we can assume that $\alpha_{R}(\Omega) \leq \epsilon_{0}$ where $\epsilon_{0}=\epsilon_{0}(N, R)$ is the constant in Theorem 3.1. Hence

$$
\operatorname{cap}_{R}(\Omega)-\operatorname{cap}_{R}\left(B_{1}\right) \geq c(N, R) \alpha_{R}(\Omega) \geq c(N, R)\left|\Omega \Delta B_{1}\right|^{2} .
$$

\section{REFERENCES}

[AAC86] N. Aguilera, H. W. Alt, and L. A. Caffarelli. An optimization problem with volume constraint. SIAM J. Control Optim., 24(2):191-198, 1986.

[AC81] H.W. Alt and L.A. Caffarelli. Existence and regularity for a minimum problem with free boundary. J. Reine Angew. Math., 325:105-144, 1981.

[ATW93] Fred Almgren, Jean E. Taylor, and Lihe Wang. Curvature-driven flows: a variational approach. SIAM J. Control Optim., 31(2):387-438, 1993.

[BDP17] Lorenzo Brasco and Guido De Philippis. Spectral inequalities in quantitative form. In Shape optimization and spectral theory, pages 201-281. De Gruyter Open, Warsaw, 2017.

[BDPV15] L. Brasco, G. De Philippis, and B. Velichkov. Faber-krahn inequalities in sharp quantitative form. Duke Math. J., 164(9):1777-1831, 2015. 
[CL12] M. Cicalese and G. P. Leonardi. A selection principle for the sharp quantitative isoperimetric inequality. Arch. Ration. Mech. Anal., 206:617-643, 2012.

[Dam02] Marc Dambrine. On variations of the shape Hessian and sufficient conditions for the stability of critical shapes. RACSAM. Rev. R. Acad. Cienc. Exactas Fís. Nat. Ser. A Mat., 96(1):95-121, 2002.

[EG15] Lawrence C. Evans and Ronald F. Gariepy. Measure theory and fine properties of functions. Textbooks in Mathematics. CRC Press, Boca Raton, FL, revised edition, 2015.

[FMP08] N. Fusco, F. Maggi, and A. Pratelli. The sharp quantitative isoperimetric inequality. Ann. Math., 168:941-980, 2008.

[FMP09] N. Fusco, F. Maggi, and A. Pratelli. Stability estimates for certain faber-krahn, isocapacitary and cheeger inequalities. Annali della Scuola Normale Superiore di Pisa-Classe di Scienze, 8:51-71, 2009.

[Fug89] B. Fuglede. Stability in the isoperimetric problem for convex or nearly spherical domains in $\mathbb{R}^{n}$. Trans. Amer. Math. Soc., 314:619-638, 1989.

[GM12] M. Giaquinta and L. Martinazzi. An Introduction to the Regularity Theory for Elliptic Systems, Harmonic Maps and Minimal Graphs, volume 11 of Lecture Notes (Scuola Normale Superiore). Edizioni della Normale, 2012.

[HHW91] R.R Hall, W.K. Hayman, and A.W. Weitsman. On asymmetry and capacity. J. d'Analyse Math., 56:87-123, 1991.

[HN92] Wolfhard Hansen and Nikolai Nadirashvili. Isoperimetric inequalities for capacities. In Harmonic analysis and discrete potential theory (Frascati, 1991), pages 193-206. Plenum, New York, 1992.

[LL97] Elliott H. Lieb and Michael Loss. Analysis, volume 14 of Graduate Studies in Mathematics. American Mathematical Society, Providence, RI, 1997.

G.D.P.: SiSSA, Via Bonomea 265, 34136 Trieste, Italy

E-mail address: guido.dephilippis@sissa.it

M.M: SiSSA, Via Bonomea 265, 34136 Trieste, Italy

E-mail address: mmarini@sissa.it

E.M: SISSA, Via Bonomea 265, 34136 Trieste, Italy

E-mail address: emukosee@sissa.it 